\title{
1 Identifying and correcting repeat-calling errors in nanopore sequencing of 2 telomeres
}

3

4

5

\author{
Kar-Tong Tan ${ }^{1,2,3}$, Michael K. Slevin ${ }^{1,4}$, Matthew Meyerson ${ }^{1,2,3,4, \#}$, Heng Li $i^{5,6, \#}$ \\ ${ }_{1}^{1}$ Department of Medical Oncology, Dana-Farber Cancer Institute, Boston, MA, USA \\ ${ }^{2}$ Cancer Program, Broad Institute of MIT and Harvard, Cambridge, MA, USA \\ ${ }^{3}$ Department of Genetics, Harvard Medical School, Boston, MA, USA \\ ${ }^{4}$ Center for Cancer Genomics, Dana-Farber Cancer Institute, Boston, MA, USA \\ ${ }^{5}$ Department of Data Sciences, Dana-Farber Cancer Institute, Boston, MA, USA \\ ${ }^{6}$ Department of Biomedical Informatics, Harvard Medical School, Boston, MA, USA \\ \#Correspondence to matthew meyerson@dfci.harvard.edu or hli@jimmy.harvard.edu
}

\section{Keywords}

Nanopore-sequencing, long-reads, telomere, basecalling 


\section{Background}

Telomeres are protective caps found on chromosomal ends, and are known to play critical roles in a wide range of biological processes and human diseases $[1,2]$. These highly repetitive structures enable cells to deal with the "end-replication problem" through the action of telomerase which adds telomeric repeats to the ends of chromosomes. In cancer, the reactivation of telomerase to drive telomere elongation is estimated to occur in as many as $90 \%$ of human cancers, and has been shown experimentally to be critical for malignant transformation [3-8]. As one ages, telomeres are also known to progressively shorten, and are thus thought to also play a central role in the process of aging [9-11]. In many organisms, telomeres are characterized by (TTAGGG) ${ }_{n}$ repeats that vary in length of between 2 and 20kb long, which are not readily resolved by short-read sequencing approaches. Given the importance of telomeres in a wide range of biological process and the technical challenges associated with their analysis using short-read sequencing, there is significant interest in applying emerging techniques like long-read sequencing to study these repetitive structures.

Long-read sequencing has emerged as a powerful technology for the study of long repetitive elements in the genome. Two main platforms, Single Molecule Real Time (SMRT) sequencing, and Nanopore sequencing, have been developed to generate sequence reads of over 10 kilobases from DNA molecules [12,13]. In SMRT Sequencing, the incorporation of DNA nucleotides is captured real time via one of four different fluorescent dyes attached to each of the four DNA bases, thereby allowing the corresponding DNA sequence to be inferred. Sequencing of the same DNA molecule multiple times in a circular manner further allows highly accurate consensus sequence of the DNA molecule to be generated in a process termed Pacific Biosciences (PacBio) High-Fidelity (HiFi) sequencing [12]. During Nanopore sequencing, the ionic current, which varies according to the DNA sequence, is measured while a single-stranded DNA molecule passes through a nanopore channel. The electrical current measurement is then converted into the corresponding DNA sequence using a deep neural network trained on a collection of ionic current profiles of known DNA sequences [13]. Notably, both platforms enable long DNA molecules of more than 10 kilo-base-pairs to be routinely sequenced and are thus highly suited for the study of long repetitive elements like telomeres.

\section{Results and discussion}

In our analysis of telomeric regions with nanopore long-read sequencing in the recently sequenced and assembled $\mathrm{CHM} 13$ sample [14,15], we surprisingly observed that telomeric regions were frequently miscalled as other types of repeats in a strand-specific manner. Specifically, although human telomeres are typically represented by (TTAGGG) $n$ repeats (Supplementary Figure 1a), these regions were frequently recorded as (TTAAAA $)_{n}$ repeats (Figure 1a,b, Supplementary Figure 1 and 2a). At the same time, when examining the reverse complementary strand of the telomeres which are represented as (CCCTAA $)_{n}$ repeats, we instead observed frequent substitution of these regions by (CTTCTT $)_{n}$ and (CCCTGG) repeats (Figure 1a,b, Supplementary Figure 1 and $\mathbf{2 b}, \mathbf{c}$ ). Notably, these artefacts were not observed on the CHM13 reference genome [14,15], or PacBio HiFi reads from the same site (Figure $1 a, b)$, suggesting that these observed repeats are artefacts of Nanopore sequencing or the base-calling process, rather than real biological variations of telomeres. Further, these repeatcalling errors could be observed on all chromosomal arms for the CHM13 sample (Supplementary Figure 1b,c), and were thus not restricted to a single chromosomal arm. The examination of each telomeric long-read also indicates that these error repeats frequently cooccur with telomeric repeats at the ends of each read (Figure 1c, Supplementary Figure 3). 
Together, our results suggest that telomeric regions are frequently misrepresented as other types of repeats in a strand-specific manner during Nanopore sequencing.

We then assessed if these errors are broadly observed in other studies or are specific to the CHM13 dataset from the Telomere-to-Telomere consortium. To assess this, we examined the previously published NA12878 and HG002 Nanopore genome sequencing datasets $[12,13,16]$. Remarkably, the same basecalling errors, TTAGGG $\rightarrow$ TTAAAA, CCCTAA $\rightarrow$ CTTCTT, and CCCTAA $\rightarrow$ CCCTGG, were similarly observed at telomeres in these datasets (Figure 1d, Supplementary Figure 4a), suggesting that these basecalling errors at telomeres are broadly observed across multiple studies. Remarkably, between $40-60 \%$ of reads at telomeric regions in these three datasets display at least one of these type of basecalling repeat artefacts for the Nanopore sequencing platform (Supplementary Figure $4 \mathbf{b}$ ), while these errors were not observed in the PacBio HiFi datasets for the same samples (Supplementary Figure 4b). Further, we also partitioned these datasets based on the sequencing platforms used to generate them, and noted that basecalling error repeats are observed across all three nanopore sequencing platforms (MinION, GridlON, PromethION) (Figure 1d, Supplementary Figure 4a). Together, these results show that these error repeats extend across nanopore sequencing datasets and sequencing platforms.

We then questioned if these error repeats are unique to specific nanopore basecallers or basecalling models. We extracted reads from chromosomal ends, and re-basecalled ionic current data of these reads using different basecallers and basecalling models. Using the production-ready basecaller Guppy5 (Oxford Nanopore Technologies), and the developmentalphase basecaller Bonito (Oxford Nanopore Technologies), we noticed that these basecalling error repeats can be readily observed across both basecallers (Figure 1e, Supplementary Figure 5 and 6). Further, these error repeats were also observed when different basecalling models were applied (Figure 1e). Significantly, we also observed that the "fast" basecalling mode in Guppy led to almost complete loss of the (CCCTAA) n strand (Figure 1e, Supplementary Figure 5a), while the "HAC" basecalling model enabled both strands to be recovered, highlighting that the basecalling model applied can affect strand-specific recovery of telomeric reads. Together, these results suggest that error repeats are observable across nanopore basecallers, and basecalling models.

To determine the cause for these repeat-calling errors, we then examined the ionic current profiles of these repeats. We thus generated ionic current profiles of these telomeric repeats and these error repeats, induced by the nanopore basecallers, using known mean current values of different 6-mers (Methods). Remarkably, we observed a high degree of similarity between current profiles between telomeric repeats and these basecalling errors (Figure 1f). Specifically, we observed that (TTAGGG) ${ }_{n}$ telomeric repeats had a high degree of similarity with the $(\text { TTAAAA })_{n}$ error repeats generated by the Bonito base-caller $($ Pearson correlation $=0.9928$, Euclidean distance $=4.9934$ ) (Supplementary Figure 7a-c). Similarly, $(\text { CCCTAA })_{n}$ current profile also showed high similarity with $(\mathrm{CCCTGG})_{\mathrm{n}}$ repeats $($ Pearson correlation $=0.9783$, Euclidean distance $=4.687$ ), and reasonably good similarity with $(\text { CTTCTT })_{n}$ repeats (Pearson correlation $=0.6411$, Euclidean distance $=19.384)$ (Supplementary Figure 7a-c).. Together, these results suggest that similarities in current profiles between repeat sequences are possible causes for repeat-calling errors at telomeric repeats.

We then examined if repeat-calling errors may extend to other repetitive sequences beyond telomeric sequences. To address this, we search for other repeat pairs with similar current 140 profiles that may be susceptible to these repeat-calling errors. We simulated and performed 
142

143

144

145

146

147

148

149

150

151

152

153

154

155

156

157

158

159

160

161

162

163

164

165

166

167

168

169

170

171

172

173

174

175

176

177

178

179

180

181

182

183

184

185

186

187

188

189

190

191

(Methods). Using similar Pearson correlation ( $\geq 0.99)$ and Euclidean distance cutoffs $(\leq 5)$ as observed for telomeric repeat errors identified in this study (Supplementary Figure 7a-c), we identified a further 2577 pairs of repeats with similar current profiles (Supplementary Table 1, Supplementary Figure 7d). For instance, we found that (TTAGGG) ${ }_{n}$ telomeric repeats also showed high similarities in current profiles with repeats with single-nucleotide substitutions like $(T T A \underline{A} G G)_{n},(T T A G \underline{A} G)_{n}$ and (TTGGGG) (Supplementary Figure 7d,e). Repeat sequences like $(\overline{G C T G C T})_{n}$ and $(\underline{A A G G C})_{n}$ that differed drastically at the sequence level, but shared similar current profiles were also observed (Supplementary Figure 7d,f). Further, we also examined the unmappable pool of $\mathrm{CHM} 13$ nanopore reads after mapping it to the $\mathrm{CHM} 13$ reference assembly. Remarkably, a significant pool of reads with long (GT) repeats were readily observed (Supplementary Figure 8). Interestingly, (GTGTGT) ${ }_{n}$ repeats were also found to have high similarities in current profiles with $(C T C T C T)_{n}$ repeats (Supplementary Figure 7d, Supplementary Table 1), suggesting that the pool of unmappable $(\mathrm{GT})_{\mathrm{n}}$ reads may include $(C T)_{n}$ repeats. Collectively, our results suggests that these basecalling error repeats may be observed at other repetitive regions, beyond telomeres.

To resolve these basecalling errors at telomeres, we then attempted to tune the nanopore basecaller by providing it with more training examples of telomeres (Figure 2a). Notably, model training was performed with a low learning rate to ensure that the majority of the model does not get affected during training while ensuring that minor adjustments in the model can be made to accurately basecall telomeres. Specifically, we tuned the deep neural network model underlying the Bonito basecaller by training it at a low learning rate with ground truth telomeric sequences extracted from the CHM13 reference genome, and current data of the corresponding reads (Methods). As two Nanopore PromethION runs were performed on the CHM13 dataset, we used the data from one run for training (run225) and tuning of the basecaller, and held out the data from the second run (run 226) for evaluation of our tuned basecaller. With this approach, we see a significant improvement in the base-calls of both the telomeres, and sub-telomeric regions on the training data and held out dataset with clearly observable decrease in errors on the chromosomal ends (Figure 2b, Supplementary Figure 9a-d). Together, our results indicate that a nanopore base-caller can be tuned to more accurately base-call telomeric regions by providing additional training examples.

As it is computationally more efficient to redo repeat-calling only for the small fraction of problematic telomeric reads rather than all reads, we developed an overall strategy to select these telomeric reads for re-basecalling with the tuned Bonito+telomeres basecaller (Figure 2c). To select telomeric reads for selective re-basecalling, we relied on an observation from the CHM13 reference genome and nanopore sequencing datasets. Specifically, we noticed that telomeric reads which maps to the ends of the CHM13 reference genome tend to show a high frequency of telomeric, or basecalling error repeats as compared to the rest of the genome (Supplementary Figure 10). We therefore utilized this observation to separate the nontelomeric reads, from the candidate telomeric reads (Figure 2c, Methods). These telomeric reads were then re-base-called with the tuned Bonito basecaller before being recombined with the pool of non-telomeric reads. Remarkably, with this strategy, we observed a significant improvement in recovery of telomeric reads with (TTAGGG) $)_{n}$ and (CCCTAA) $n$ repeats (from 384 to 476 TTAGGG and 373 to 686 CCCTAA reads) (Figure 2d). At the same time, a sharp reduction of these basecalling repeat errors was also observed (151 to 17 TTAAAA reads, 561 to 48 CTTCTT reads, and 337 to 20 CCCTGG reads) (Figure 2d). Together, these results suggests that our "selective tuning" approach for fixing basecalling errors at telomeres can improve recovery of telomeric reads while reducing telomeric basecalling repeat artefacts. 
192 We further evaluated our approach for possible impact on overall basecalling accuracy. While a reduction in global basecalling accuracy was observed $(\sim 1-2 \%)$ when our tuned basecaller was directly applied to the full dataset, caused likely by miscalling of endogenous (CTTCTT $)_{n}$ genomic repeats as (CCCTAA $)_{n}$, this loss of global basecalling accuracy could be avoided by applying our basecaller to telomeric reads alone. Concordant with this, we did not observe changes in overall basecalling accuracy with our telomere-selective tuning approach (Figure 2e). These results indicate that our telomere-selective tuning approach has negligible impact on basecalling accuracy for the rest of the genome.

\section{Conclusion}

In this study, we showed that basecalling errors can be widely observed at telomeric regions across nanopore datasets, sequencing platforms, basecallers, and basecalling models. We further showed that these strand-specific basecalling errors were likely induced by similarities in current profiles between different repeat types. To resolve these basecalling errors at telomeres, we devised an overall strategy to re-basecall telomeric reads using a tuned nanopore basecaller. More broadly, our study highlights the importance of verifying nanopore basecalls in long, repetitive and poorly defined regions of the genome. For instance, this can be done either with an orthogonal platform, or at a minimum by ensuring nanopore basecalls between opposite strands are concordant. In the future, we anticipate that further improvements in the nanopore basecaller or basecalling model as demonstrated in this study will potentially lead to the reduction or elimination of these basecalling artefacts. 


\section{Methods}

\section{Nanopore and PacBio Datasets}

Nanopore and PacBio HiFi datasets for the CHM13 sample were downloaded directly from the telomere-to-telomere consortium (https://github.com/marbl/CHM13)

Nanopore dataset for GM12878 was obtained from the Nanopore WGS consortium (https://github.com/nanopore-wgs-consortium/NA12878/blob/master/Genome.md). PacBio HiFi dataset for GM12878 was obtained from the repository at the SRA database (SRP194450), and downloaded from the lollowing (https://www.ebi.ac.uk/ena/browser/view/SRR9001768?show=reads)

The HG002 PacBio HiFi and Nanopore datasets were downloaded from the Human Pangenome Reference Consortium (https://github.com/humanpangenomics/HG002 Data Freeze v1.0). Specifically, the HG002 Data Freeze (v1.0) recommended downsampled data mix was downloaded. The PacBio HiFi dataset corresponds to $34 \mathrm{X}$ coverage of Sequel II System with Chemistry 2.0. The Nanopore dataset corresponds to $60 x$ coverage of unsheared sequencing from 3 PromethION flow cells from Shafin et al [17].

\section{Extraction of candidate telomeric reads}

Telomeric reads were extracted by mapping all reads to the CHM13 draft genome assembly (v1.0) obtained from the telomere-to-telomere consortium using Minimap2 (version 2.17-r941). Subsequent to that, reads that mapped to within 10 kilobasepairs of the start and end of each autosome and X-chromosome were then extracted using SAMtools (version 1.10).

\section{Co-occurrence matrix}

Candidate PacBio HiFi and Nanopore telomeric reads were first extracted as described above, and then converted into the FASTA format using SAMtools (version 1.10). Subsequent to that, custom Python scripts were used to assess if each of the reads contain at least four consecutive counts of the repeat sequence of interest (e.g. (TTAGGG) $)_{4}$ ). This information is then used to generate a pair-wise correlation matrix as depicted with $\mathrm{R}$ in the main text.

\section{Basecalling of nanopore data with different basecallers and basecalling models}

Basecalling of Nanopore data was done using Guppy (Version 4.4.2), Guppy (Version 5.0.16) and Bonito v0.3.5 (commit d8ae5eeb834d4fa05b441dc8f034ee04cb704c69). For Guppy4, four different basecalling models were applied (guppy_dna_r9.4.1_450bps_fast, guppy_dna_r9.4.1_450bps_hac, guppy_dna_r9.4.1_450bps_prom_fast, guppy_dna_r9.4.1_450bps_prom_hac). For Guppy 5, six different basecalling models were applied (dna_r9.4.1_450bps_fast, dna_r9.4.1_450bps_hac, dna_r9.4.1_450bps_sup, dna_r9.4.1_450bps_fast_prom, dna_r9.4.1_450bps_hac_prom, dna_r9.4.1_450bps_sup_prom) For Bonito, the v1, v2, v3, v3.1 and default basecalling models were applied.

\section{Current profiles for different repeat sequences}

The mean current level for different k-mers sequenced by Nanopore sequencing was obtained from the k-mer models published by Oxford Nanopore (https://github.com/nanoporetech/kmer models/tree/master/r9.4 180mv 450bps 6mer).

Circular permutations of each 6-mer of interest was generated, and their corresponding mean current level extracted from the k-mer models. The current profiles for each of the indicated repeat sequences were then plotted and depicted in the figure. 


\title{
Pairwise comparison of all possible k-mers
}

Current profile for each 6-mer repeat sequence was generated using the published k-mer models as described above. Pairwise comparisons of all possible 6-mer repeat current profiles was then performed $(8,386,560$ pairs in total). A corresponding (i) Pearson correlation value, (ii) mean-centered Euclidean distance, and (iii) mean current difference for each pair of 6-mer repeat current profiles were then generated. Pairs of repeats with a Pearson correlation value $\geq$ 0.99 and Euclidean distance $\leq 5$ were selected as putative repeat pairs that can be miscalled.

\section{Tuning of bonito model}

The default model from Bonito v0.3.5 (commit d8ae5eeb834d4fa05b441dc8f034ee04cb704c69) was used as the base model for model tuning. The training dataset needed for the training process was generated from the telomeric reads from a PromethION run in the $\mathrm{CHM} 13$ dataset (run225). More broadly, we then generate the training dataset by matching the current profiles from the Nanopore run to ground truth sequences that we extracted from the CHM13 draft reference genome assembly (v1.0) using custom written code.

Specifically, these telomeric reads were first basecalled using the initial Bonito basecalling model, and then mapped back to the CHM13 draft reference genome assembly (v1.0). This allowed each telomeric read to be properly assigned to its corresponding chromosomal arm with its sub-telomeric sequence. Nonetheless, as the telomeric region of the same read could not be properly mapped to the telomeric repeats due to the repeat errors, there was difficulty in assigning the nanopore current data to the correct ground truth sequences in the reference genome. As such, the presume length of sequences to extract was estimated using the basecalling repeat error sequences, and the same length of sequences were then extracted from the CHM13 reference genome to serve as ground truth sequences. With this idea and with custom Perl script, we were able to generate a set of ground truth sequences and signals for model tuning. These data were then formatted into the corresponding python objects required by the Bonito basecaller with custom Python scripts. Using the tune function in Bonito and with our prepared training dataset, we were then able to train the basecaller to convergence.

\section{Selective application of tuned basecaller to telomeric reads}

We applied our tuned basecaller by first extracting candidate telomeric reads for re-basecalling. This was done by enumerating the total 3-mer telomeric (TTAGGG, CCCTAA) and repeat artefact count (TTAAAA, CTTCTT, CCCTGG) on each read. Reads with at least 10 total counts of these repeats were isolated and their readnames noted. These reads were then excluded from the total pool of reads via their readnames, and basecalled separately using our tuned basecaller using the fast5 data of these reads. Following basecalling with the tuned basecaller, these reads were then recombined with the main pool of reads.

\author{
Abbreviations \\ PacBio: Pacific Biosciences \\ SMRT: Single Molecule Real Time
}




\section{Author information} Kar-Tong Tan, Michael K. Slevin, Matthew Meyerson

Cancer Program, Broad Institute of MIT and Harvard, Cambridge, MA, USA

Kar-Tong Tan, Matthew Meyerson

Department of Genetics, Harvard Medical School, Boston, MA, USA

Kar-Tong Tan, Matthew Meyerson

Center for Cancer Genomics, Dana-Farber Cancer Institute, Boston, MA, USA

Michael K. Slevin, Matthew Meyerson

Department of Data Sciences, Dana-Farber Cancer Institute, Boston, MA, USA

Heng Li

Department of Biomedical Informatics, Harvard Medical School, Boston, MA, USA

Heng Li

\section{Author contributions}

K.T.T. and M.S. identified issues with Nanopore sequencing of telomeres, and discovered basecalling errors at telomeric regions. K.T.T. evaluated basecalling errors in Nanopore sequencing datasets, and designed the overall approach for correcting basecalling errors at telomeric regions with inputs from H.L. and M.M. K.T.T. wrote the initial draft of the manuscript with inputs from H.L. and M.M. M.M. and H.L. jointly supervised the work. All authors read, revised, and approved the submission of the manuscript.

\section{Corresponding authors}

347 (hli@jimmy.harvard.edu). 


\section{Declarations}

Ethics approval and consent to participate

Not applicable.

\section{Consent for publication}

Not applicable.

\section{Availability of data and materials}

Source code to apply and retrain the bonito bascalling model for telomeric region can be found at the following link: https://github.com/ktan8/nanopore telomere basecall/.

The tuned bonito basecalling model can be downloaded from

https://zenodo.org/api/files/86cb9586-300f-493d-b9c4-

0ab2f2848e3c/chm13 nanopore trained run225.zip. A comprehensive version of

Supplementary Table 1 with all possible pairs of k-mers can be found at

https://zenodo.org/api/files/86cb9586-300f-493d-b9c40ab2f2848e3c/all comparisions.similar profile.txt.zip.

\section{Competing interests}

H.L. is a consultant of Integrated DNA Technologies and on the SAB of Sentieon, Innozeen and BGI. M.M. has a patent for EGFR mutations for lung cancer diagnosis issued, licensed, and with royalties paid from LabCorp and a patent for EGFR inhibitors pending to Bayer; and was a founding advisor of, consultant to, and equity holder in Foundation Medicine, shares of which were sold to Roche.

\section{Funding}

K.T.T. is supported by a PhRMA Foundation Informatics Fellowship, and a NUS Development Grant from the National University of Singapore. M.M. is supported by an American Cancer Society Research Professorship. This work was supported by grants from the National Human Genome Research Institute (NHGRI) (Grant Nos. R01 HG010040, U01 HG010961, and U41 HG010972 to H.L.), and the National Cancer Institute (Grant No. R35 CA197568 to M.M.).

\section{Acknowledgements}

We would like to thank all members of the H.L. and M.M. labs for helpful comments and discussions. We would also like to thank the Telomere-to-Telomere consortium for generating the $\mathrm{CHM} 13$ datasets used in this study. 


\section{References}

1. Shay JW, Wright WE. Telomeres and telomerase: three decades of progress. Nat Rev Genet. 2019;

2. Turner K, Vasu V, Griffin D. Telomere Biology and Human Phenotype. Cells. 2019;

3. Li Y, Tergaonkar V. Noncanonical functions of telomerase: Implications in telomerasetargeted cancer therapies. Cancer Res. 2014.

4. Kim NW, Piatyszek MA, Prowse KR, Harley CB, West MD, Ho PLC, et al. Specific association of human telomerase activity with immortal cells and cancer. Science (80- ). 1994;

5. Meyerson M, Counter CM, Eaton EN, Ellisen LW, Steiner P, Caddle SD, et al. hEST2, the putative human telomerase catalytic subunit gene, is up- regulated in tumor cells and during immortalization. Cell. 1997;

401 6. Kolquist KA, Ellisen LW, Counter CM, Meyerson M, Tan LK, Weinberg RA, et al. Expression 402 of TERT in early premalignant lesions and a subset of cells in normal tissues. Nat Genet. 1998;

403 7. Li Y, Tergaonkar V. Telomerase reactivation in cancers: Mechanisms that govern 404 transcriptional activation of the wild-type vs. mutant TERT promoters. Transcription. 2016.

405 8. Yuan X, Larsson C, Xu D. Mechanisms underlying the activation of TERT transcription and telomerase activity in human cancer: old actors and new players. Oncogene. 2019. 9. Shay JW. Telomeres and aging. Curr. Opin. Cell Biol. 2018. 10. Aubert G, Lansdorp PM. Telomeres and aging. Physiol. Rev. 2008. 11. Shammas MA. Telomeres, lifestyle, cancer, and aging. Curr Opin Clin Nutr Metab Care. 2011;

411 12. Wenger AM, Peluso P, Rowell WJ, Chang PC, Hall RJ, Concepcion GT, et al. Accurate circular consensus long-read sequencing improves variant detection and assembly of a human genome. Nat Biotechnol. 2019;

13. Jain M, Koren S, Miga KH, Quick J, Rand AC, Sasani TA, et al. Nanopore sequencing and assembly of a human genome with ultra-long reads. Nat Biotechnol. 2018;

14. Miga KH, Koren S, Rhie A, Vollger MR, Gershman A, Bzikadze A, et al. Telomere-totelomere assembly of a complete human $\mathrm{X}$ chromosome. bioRxiv. 2019;

15. Logsdon GA, Vollger MR, Hsieh PH, Mao Y, Liskovykh MA, Koren S, et al. The structure, function and evolution of a complete human chromosome 8. Nature. 2021;

16. Zook JM, Catoe D, McDaniel J, Vang L, Spies N, Sidow A, et al. Extensive sequencing of seven human genomes to characterize benchmark reference materials. Sci Data. 2016;

17. Shafin K, Pesout T, Lorig-Roach R, Haukness M, Olsen HE, Bosworth C, et al. Nanopore sequencing and the Shasta toolkit enable efficient de novo assembly of eleven human genomes. Nat Biotechnol. 2020;38. 
bioRxiv preprint doi: https://doi.org/10.1101/2022 01.11.475254 this version posted January 12,2022 . The copyright holder for this preprint (which was not certified by peer review) is the author/funder, who has granted bioRxiv a license to display the preprint in perpetuity. It is made available under aCC-BY-NC-ND 4.0 International license.

\section{Figure 1}

a

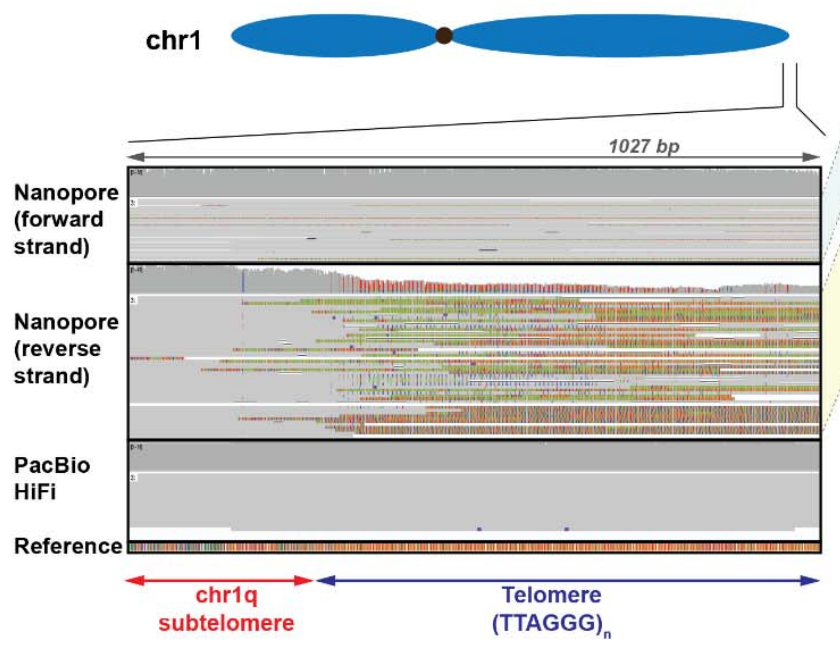

d

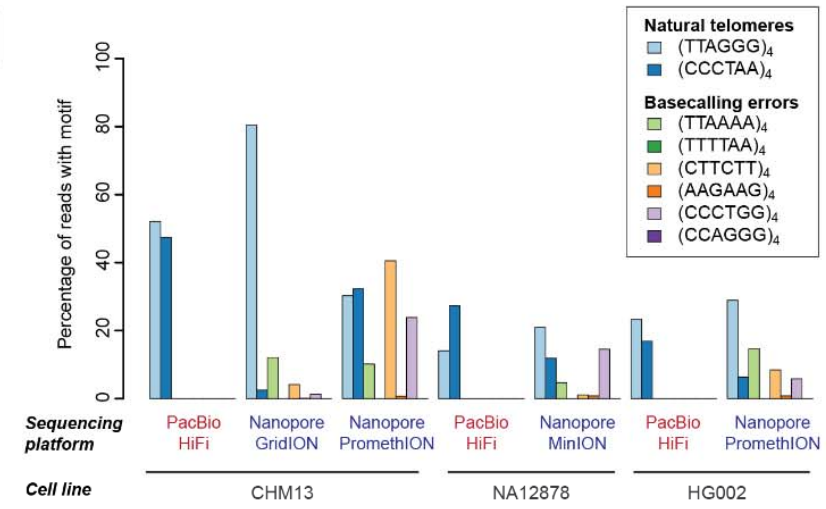

$\mathbf{e}$

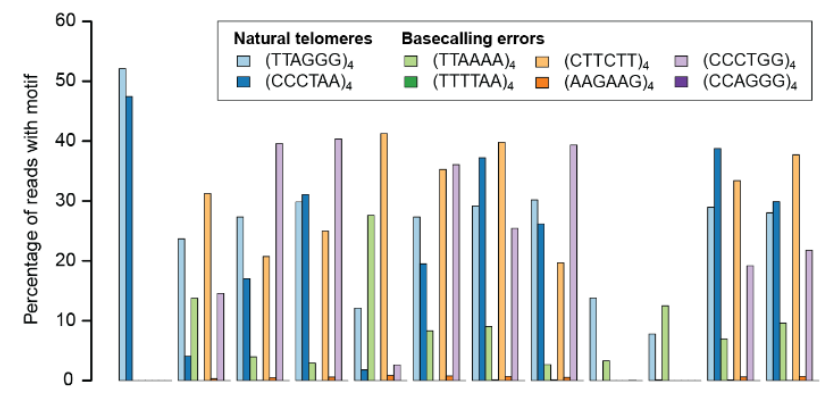

Basecalling model

Basecaller

Sequencing

platform
plancing

$\begin{array}{lllllll}\text { Fast HAC SUP Prom- Prom- Prom- Default } & \text { v1 } & \text { v2 } & \text { v3 } & \text { v3.1 }\end{array}$

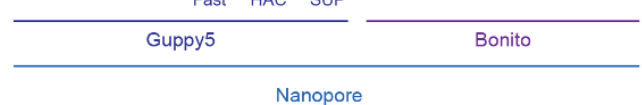

b

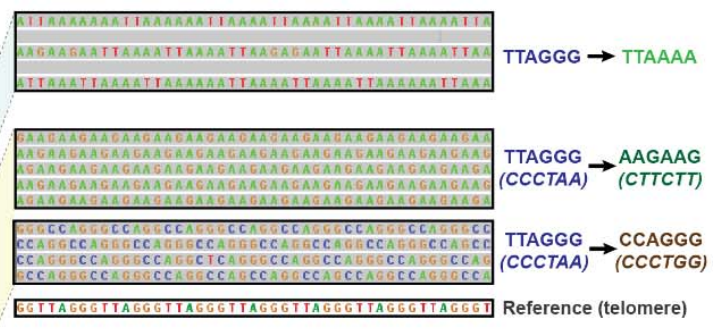

C
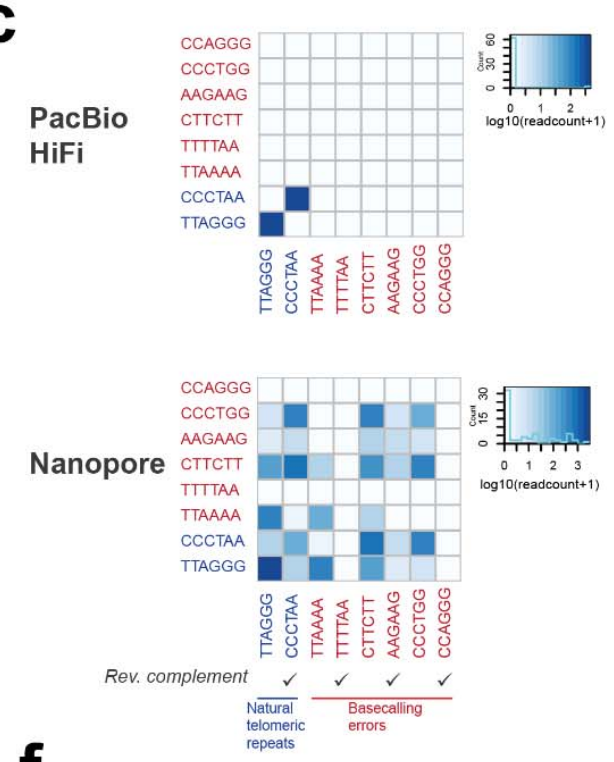

f
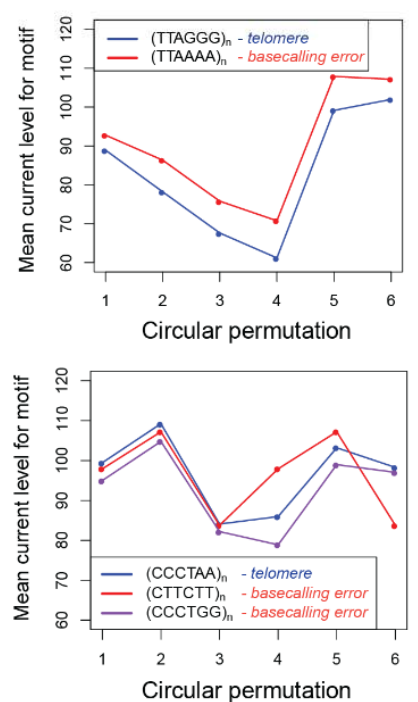

Figure 1 Strand-specific Nanopore basecalling errors are pervasive at telomeres. $(\mathbf{a}, \mathbf{b})$ IGV screenshot illustrating the three types of basecalling errors found on the forward and reverse strands of telomeres for Nanopore sequencing. (TTAGGG) $n$ on the forward strand of Nanopore sequencing data was basecalled as $(\text { TTAAAA })_{n}$ while $(\text { CCCTAA })_{n}$ on the reverse 
433 strand was basecalled as (CTTCTT $)_{n}$ and (CCCTGG $)_{n}$. PacBio HiFi data generated from the same cell line (CHM13) is depicted as a control. Reference genome indicated in the plot corresponds to the chm13 draft genome assembly (v1.0). (c) Co-occurrence heatmap illustrating the frequency of co-occurrence of repeats corresponding to natural telomeres, or to basecalling errors in PacBio HiFi and Nanopore long-reads found at chromosomal ends (within $10 \mathrm{~kb}$ of annotated end of the reference genome). Diagonal of co-occurrence matrix represents counts of long-reads with only a single type of repeats observed. (d) Basecalling errors at telomeres are observed across different nanopore datasets and sequencing platforms. (e) Basecalling errors at telomeres are observed different nanopore basecallers and basecalling models. Guppy5 and the Bonito basecallers, and different bascalling models for each bascaller, were used to basecall telomeric reads in the CHM13 PromethION dataset (reads that mapped to flanking $10 \mathrm{~kb}$ regions of the $\mathrm{CHM} 13$ reference genome). (f) Basecalling errors share similar nanopore current profiles as telomeric repeats. Current profiles for telomeric and basecalling error repeats were plotted based on known mean current profiles for each k-mer (Methods). 


\section{Figure 2}

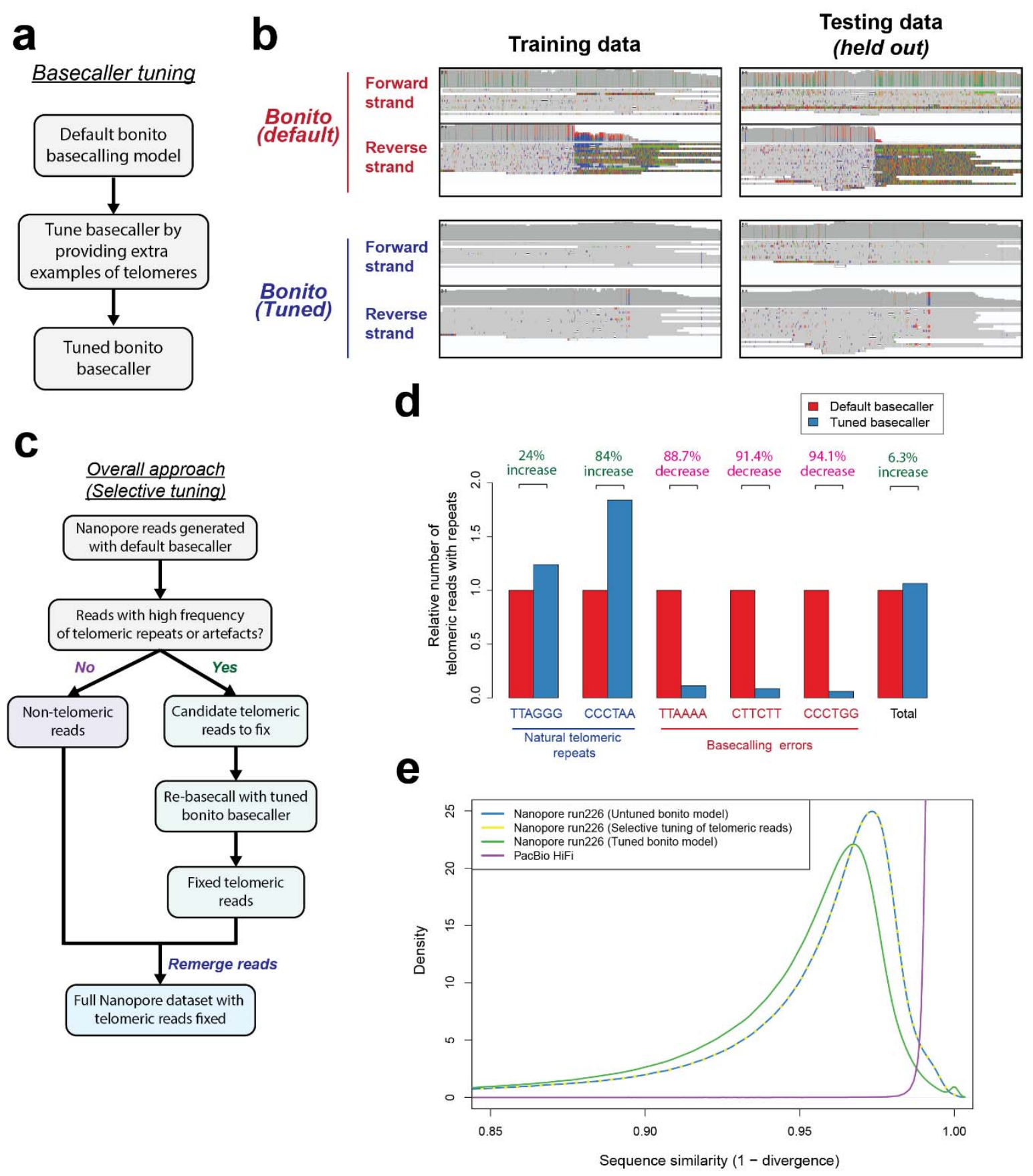

Figure 2 Selective re-basecalling of telomeric reads resolves basecalling errors at telomeres. (a) Approach for tuning the bonito basecalling model for improving basecalls at telomeres. (b) Tuned bonito basecalling model leads to improvement in basecalls at telomeric regions. IGV screenshots of telomeric region (chr2q) in the $\mathrm{CHM13}$ dataset basecalled using the default bonito basecaller, and the tuned bonito basecalling model is as depicted. (c) Overall approach for selecting and fixing telomeric reads in nanopore sequencing datasets. Telomeric reads are selected (Methods), and rebasecalled using the tuned bonito basecalling model. (d) The selective tuning approach leads to improved recovery of telomeric reads, and decrease in 
458 the number of reads with basecalling artefacts. Evaluation was performed on the held out test 459 dataset (run226). (e) The 'selective basecalling' approach leads to little detected negative 460 impact on basecalling of other genomic regions. The sequence similarity of all reads to the 461 reference genome for three approaches for basecalling of nanopore reads was evaluated. They 462 are applying the default bonito basecalling model to all reads (untuned bonito model), applying 463 the tuned bonito basecalling model to all reads (tuned bonito model), and applying the tuned 464 bonito basecalling model selectively to telomeric reads only (selective tuning of telomeric reads). 465 The density plot depicts the sequence similarity of each read against the CHM13 reference 466 genome as assessed using minimap2. 


\section{Supplementary Figure 1}

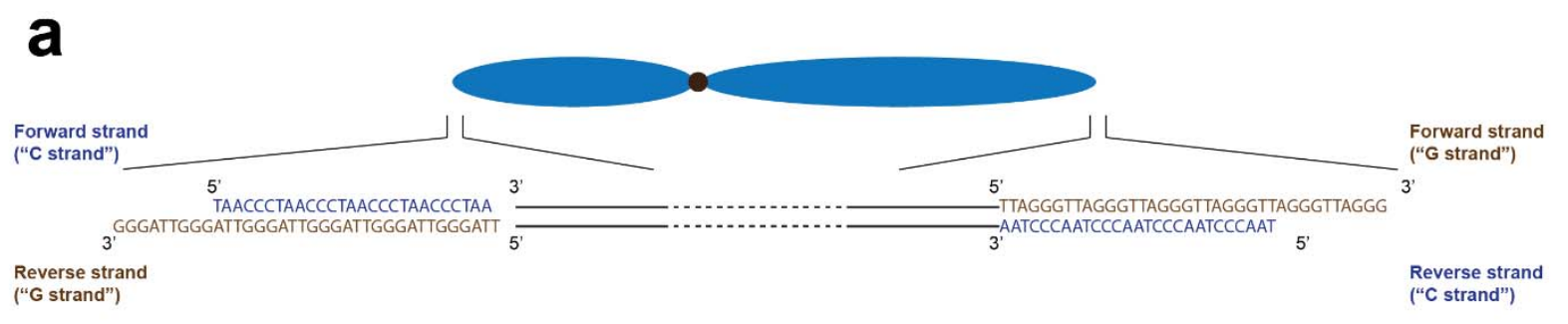

b
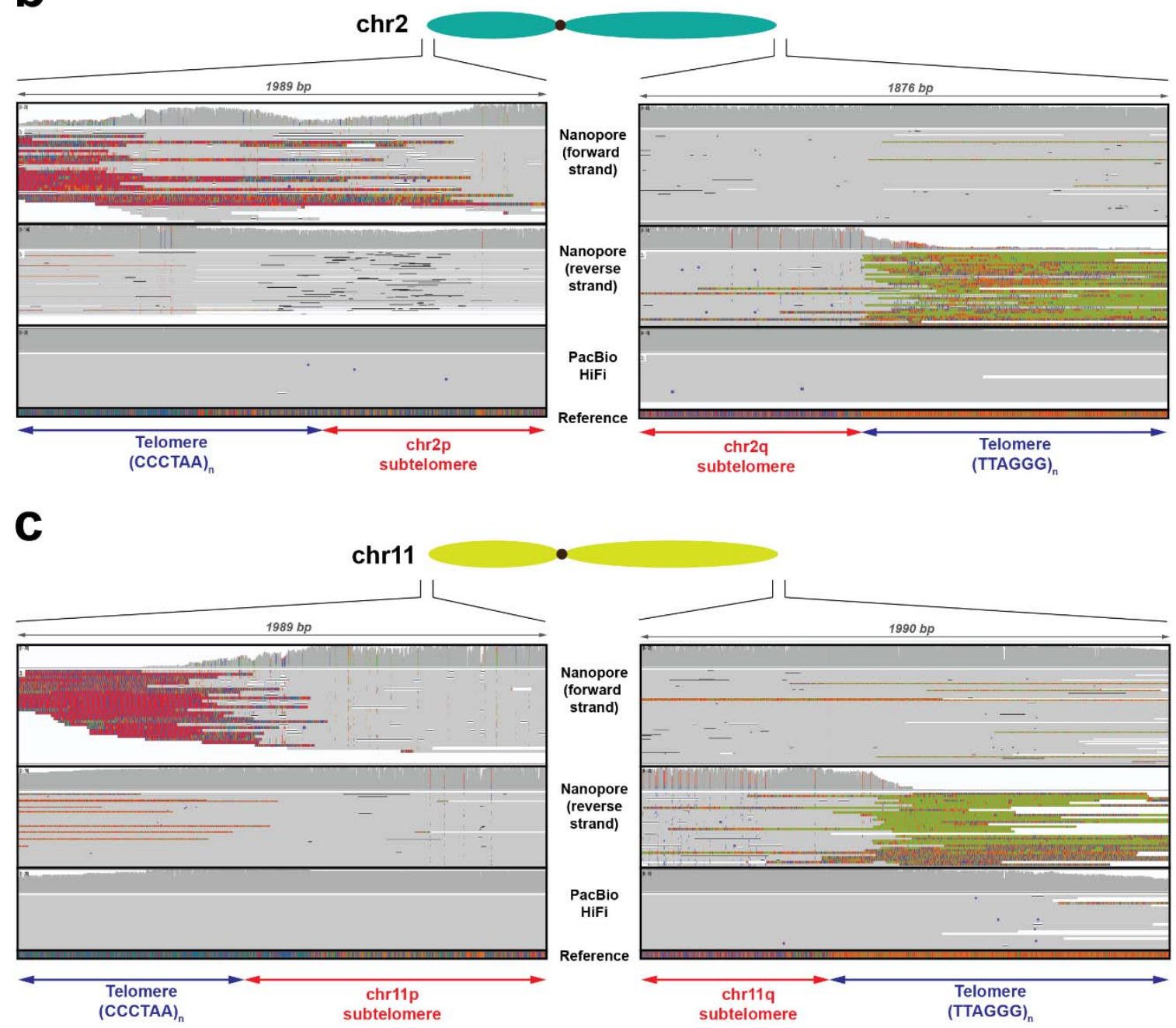

Supplementary Figure 1 Additional screenshots of basecalling repeat errors found on different chromosomal arms. (a) Schematic depicting sequence and orientation of telomeric repeat sequences on the p-arms (arm on the left in the schematic) and q-arms (arm on the right of the schematic) of a chromosome. Note that the forward strand for the arm on the left, and reverse strand for the arm on the right are "C-rich strands" and characterized by (CCCTAA)n repeats in a 5'-to-3' direction. Also note that the reverse strand for the arm on the left, and forward strand for the arm on the right are "G-rich strands" and characterized (TTAGGG) 
bioRxiv preprint doi: https://doi.org/10.1101/2022.01.11.475254; this version posted January 12, 2022. The copyright holder for this preprint (which was not certified by peer review) is the author/funder, who has granted bioRxiv a license to display the preprint in perpetuity. It is made available under aCC-BY-NC-ND 4.0 International license.

477 repeats in a 5'-to-3' direction. (b-c) Screenshots depicting additional representative examples of 478 chromosomal arms with basecalling error repeats. These are (b) chromosome 2 and (c) 479 chromosome 11. Screenshots were extracted from the Integrative Genomics Viewer for the 480 CHM13 long-read dataset mapped against the CHM13 reference genome. Related to Figure 1a.

481 
bioRxiv preprint doi: https://doi.org/10.1101/2022.01.11.475254; this version posted January 12, 2022. The copyright holder for this preprint (which was not certified by peer review) is the author/funder, who has granted bioRxiv a license to display the preprint in perpetuity. It is made available under aCC-BY-NC-ND 4.0 International license.

\section{Supplementary Figure 2}

a

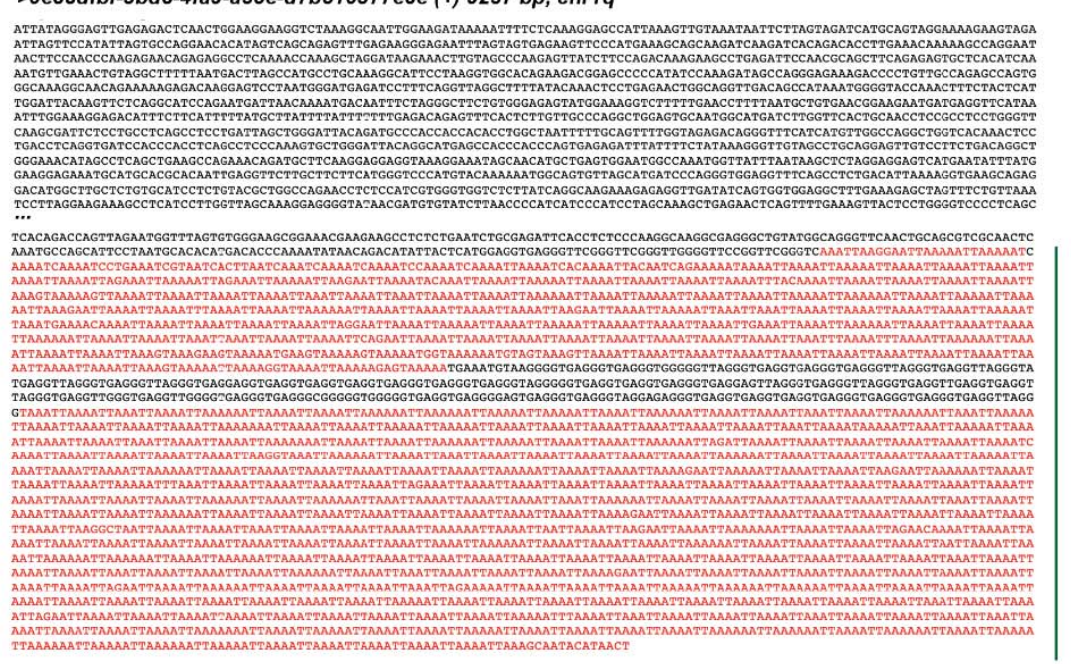

TTAGGG $\rightarrow$ TTAAAA

b

$>2 \mathrm{e} 9951 c 6-e 9 d d-4 c 87-8470-078 b 4 c b f f d 84$ (-) 42471 bp, chr1q
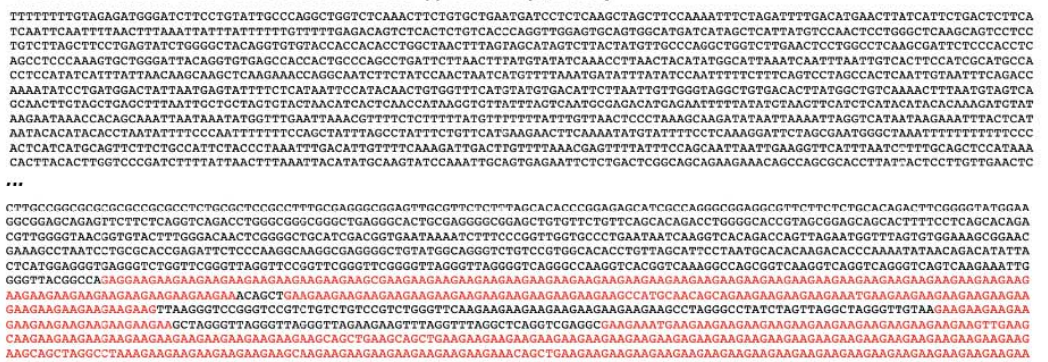

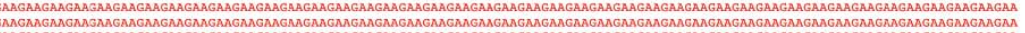

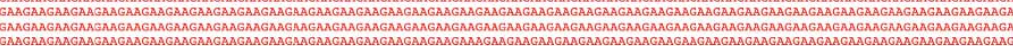

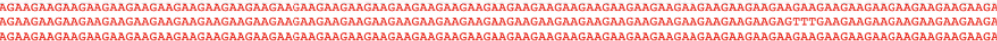

TTAGGG
$($ CCCTAA) $\rightarrow$ AAGAAG

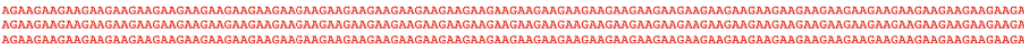

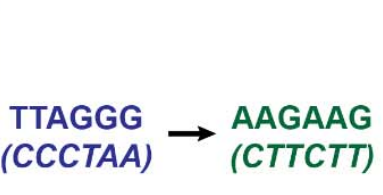
AGAAGAAGAAGAAGAAGAAGAGGAGGAAGA

C >5270195c-c854-4c76-850c-a4ec17090fdd (-) 3515 bp, chr1q

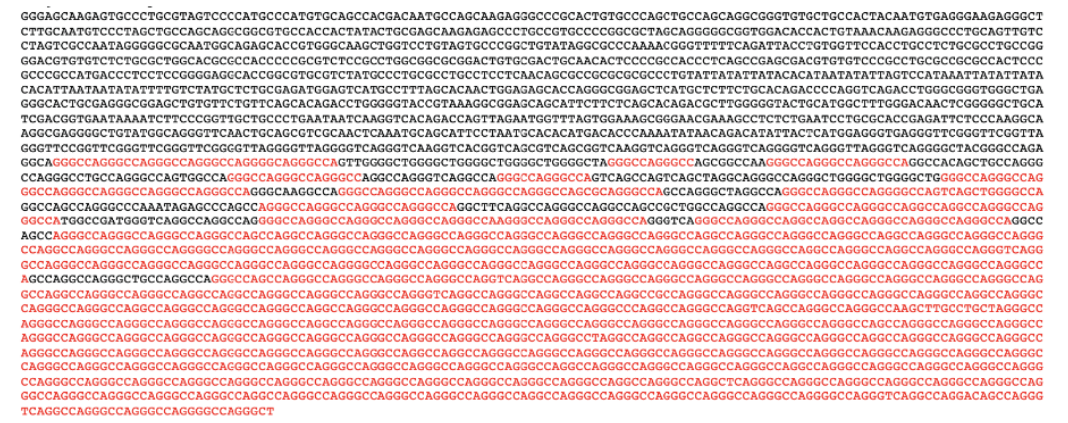

TTAGGG
$($ CCCTAA) $\rightarrow$ (CCAGGG

Supplementary Figure 2 Examples of long-reads with three types of basecalling error repeats found at telomeres. (a-c) Sequences and readnames of representative long-reads with the three reported types of basecalling error repeats are as depicted. The region with the 
bioRxiv preprint doi: https://doi.org/10.1101/2022.01.11.475254; this version posted January 12,2022 . The copyright holder for this preprint (which was not certified by peer review) is the author/funder, who has granted bioRxiv a license to display the preprint in perpetuity. It is made available under aCC-BY-NC-ND 4.0 International license.

$488(\mathrm{CCCTAA})_{\mathrm{n}}$ to $(\mathrm{CCCTGG})_{n}$. Note that (b) and (c) represents the reverse complementary 489 sequence of the actual nanopore long-read sequence. Also note that the repeats were found on 490 the end of each read as expected given that telomeric repeats are typically found on the end of 491 the chromosomes.

492 


\section{Supplementary Figure 3}

a

CCAGGG

CCCTGG

AAGAAG

CTTCTT

TTTTAA

TTAAAA

CCCTAA

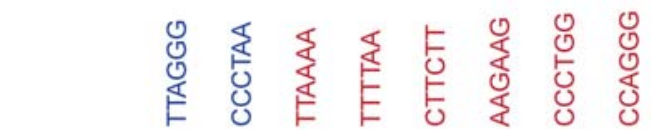

C

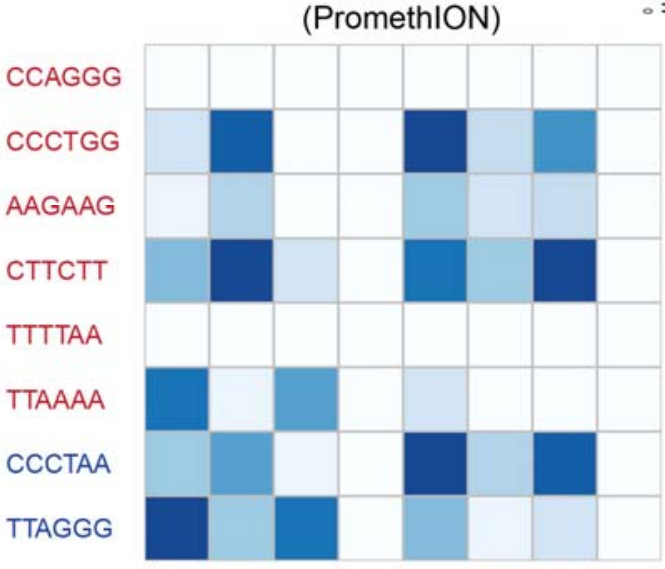

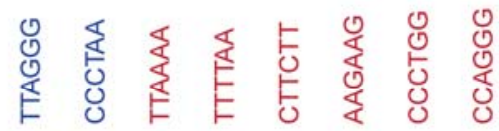

$$
\begin{aligned}
& \text { Natural } \\
& \text { telomeric } \\
& \text { repeats }
\end{aligned}
$$

TTAGGG

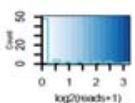

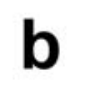
CCAGGG CCCTGG AAGAAG СTTCTT TTTTAA TTAAAA CCCTAA TTAGGG

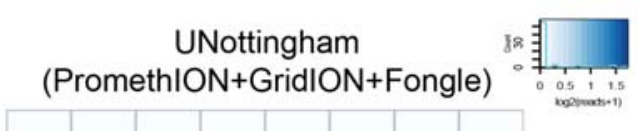
occurrence of telomeric repeats and basecalling errors for the CHM13 Nanopore dataset generated at different sites. These are (a) National Human Genome Research Institute (NHGRI), (b) University of Nottingham (UNottingham), (c) University of California, Davis (UCDavis) and (d) University of Washington (UWashington). The sequencing platforms used for 500 


\section{Supplementary Figure 4}

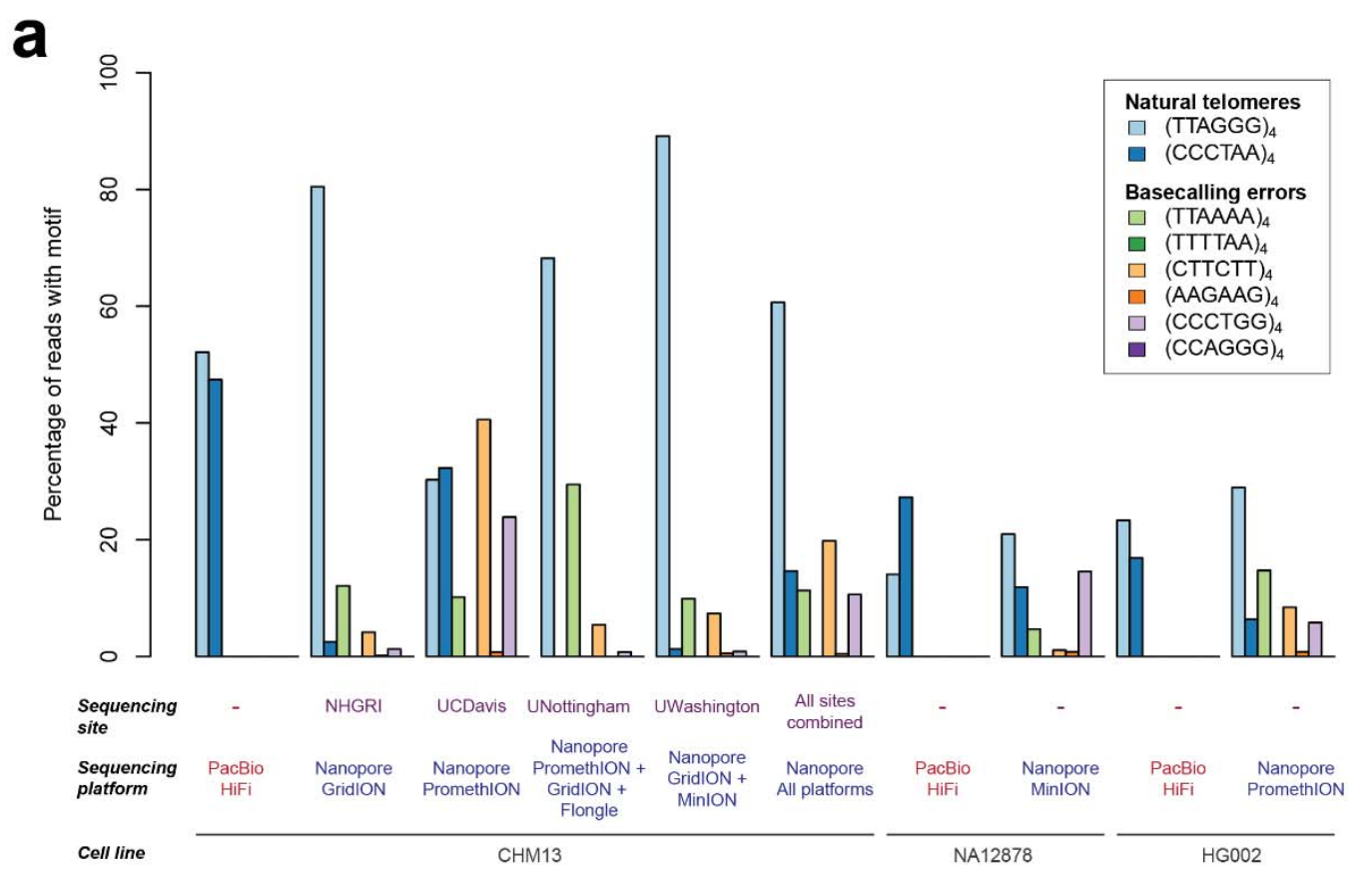

b
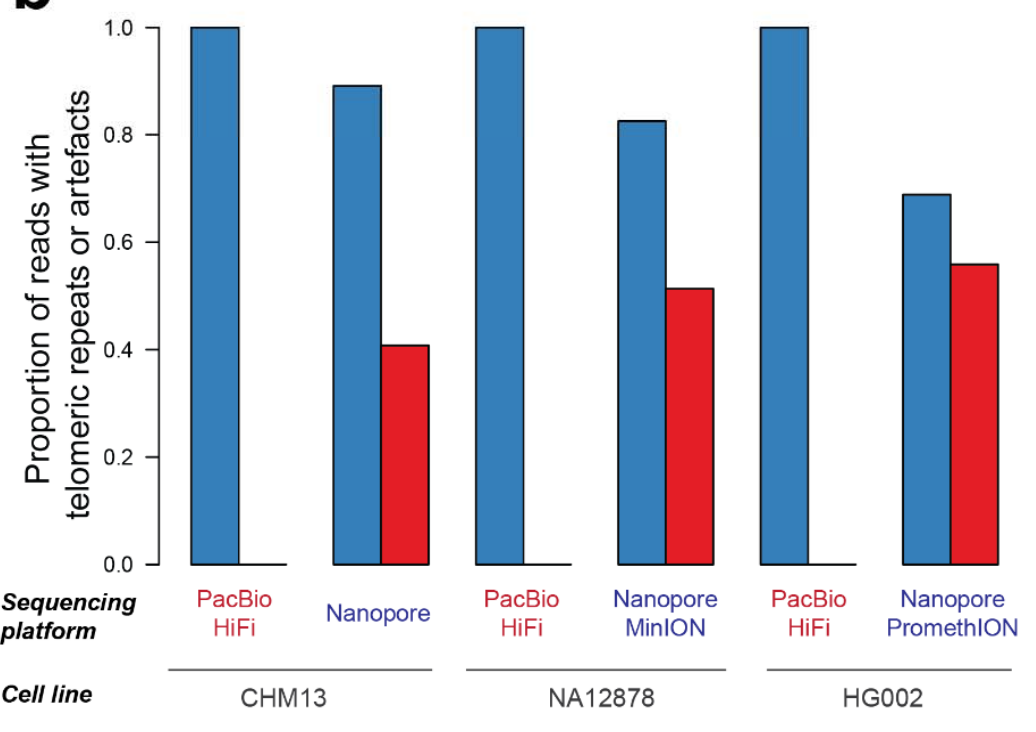

Supplementary Figure 4 Frequency of telomeric repeat errors in different Nanopore sequencing dataset and sequencing platforms. (a) Frequency of basecalling error repeats on three different cell lines generated by different Nanopore sequencing platforms. This figure is an extension Figure 1d. (b) Aggregated fraction of basecalling error repeats for different cell lines and datasets. 


\section{Supplementary Figure 5}

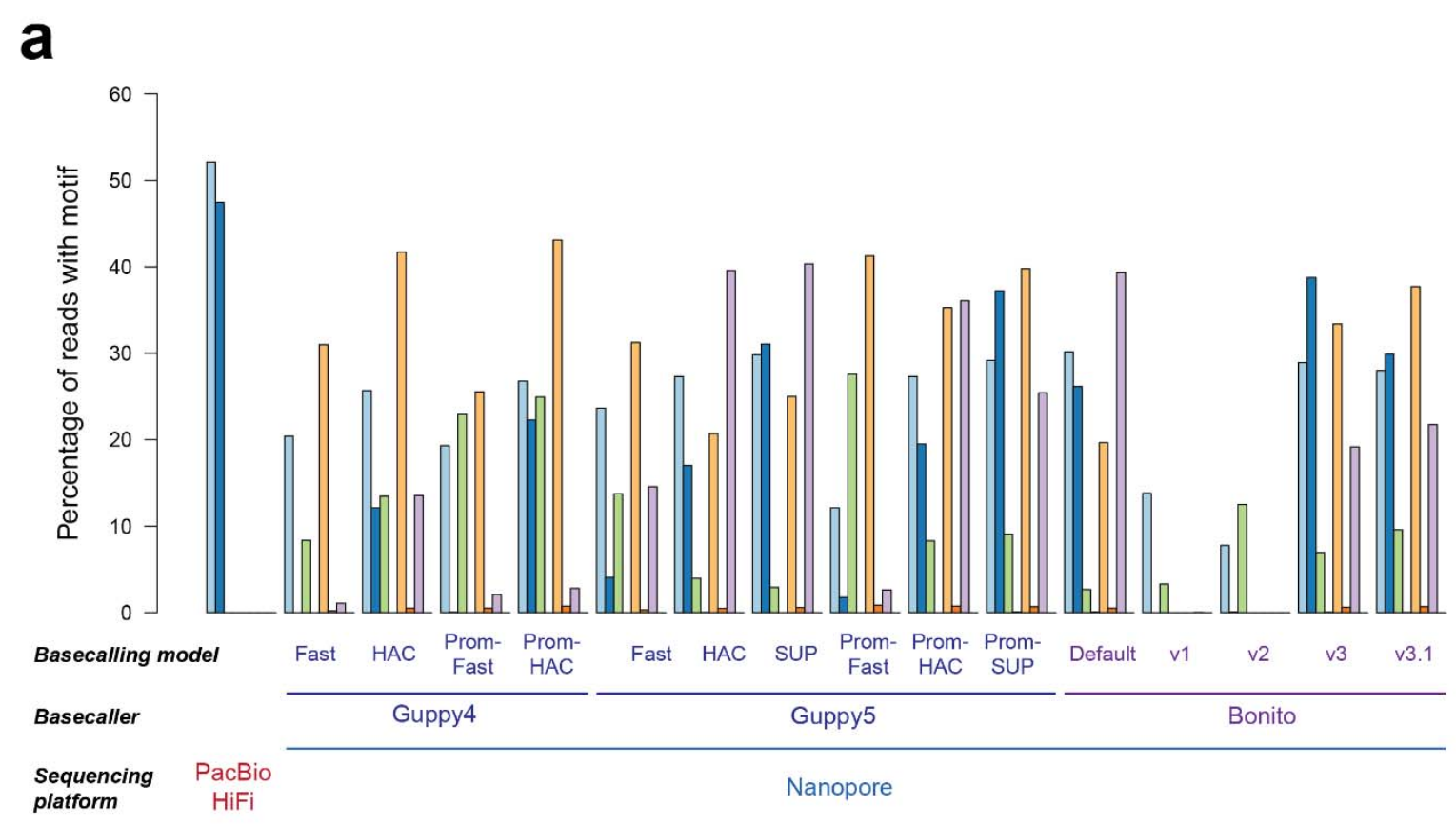

b

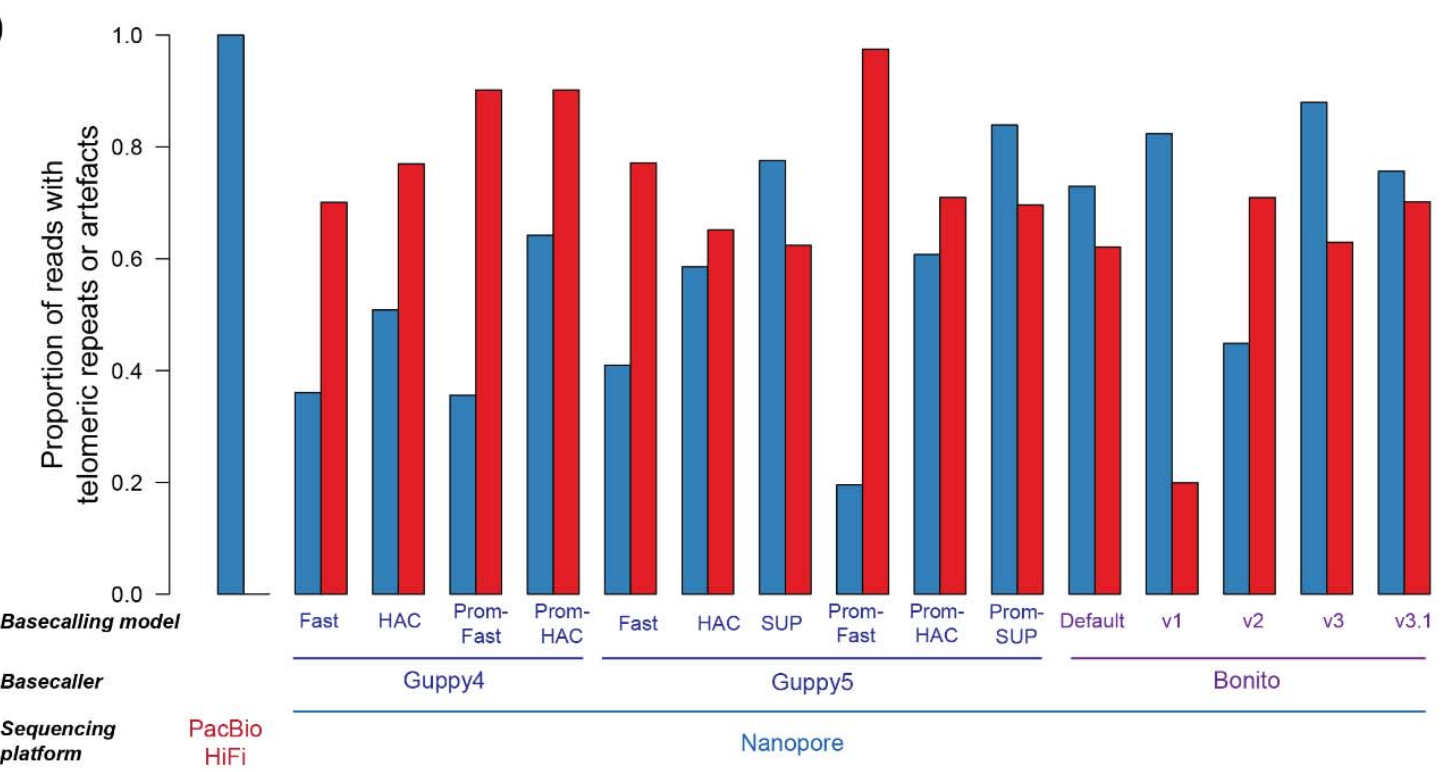

511 Supplementary Figure 5 Frequency of telomeric repeat errors in different Nanopore basecallers. (a) Frequency of basecalling error repeats for different basecallers (Guppy4, Guppy5 and Bonito) and basecalling models. This figure is an extension of Figure 1e. (b) Aggregated fraction of basecalling error repeats for different basecallers and basecalling models. 


\section{Supplementary Figure 6}
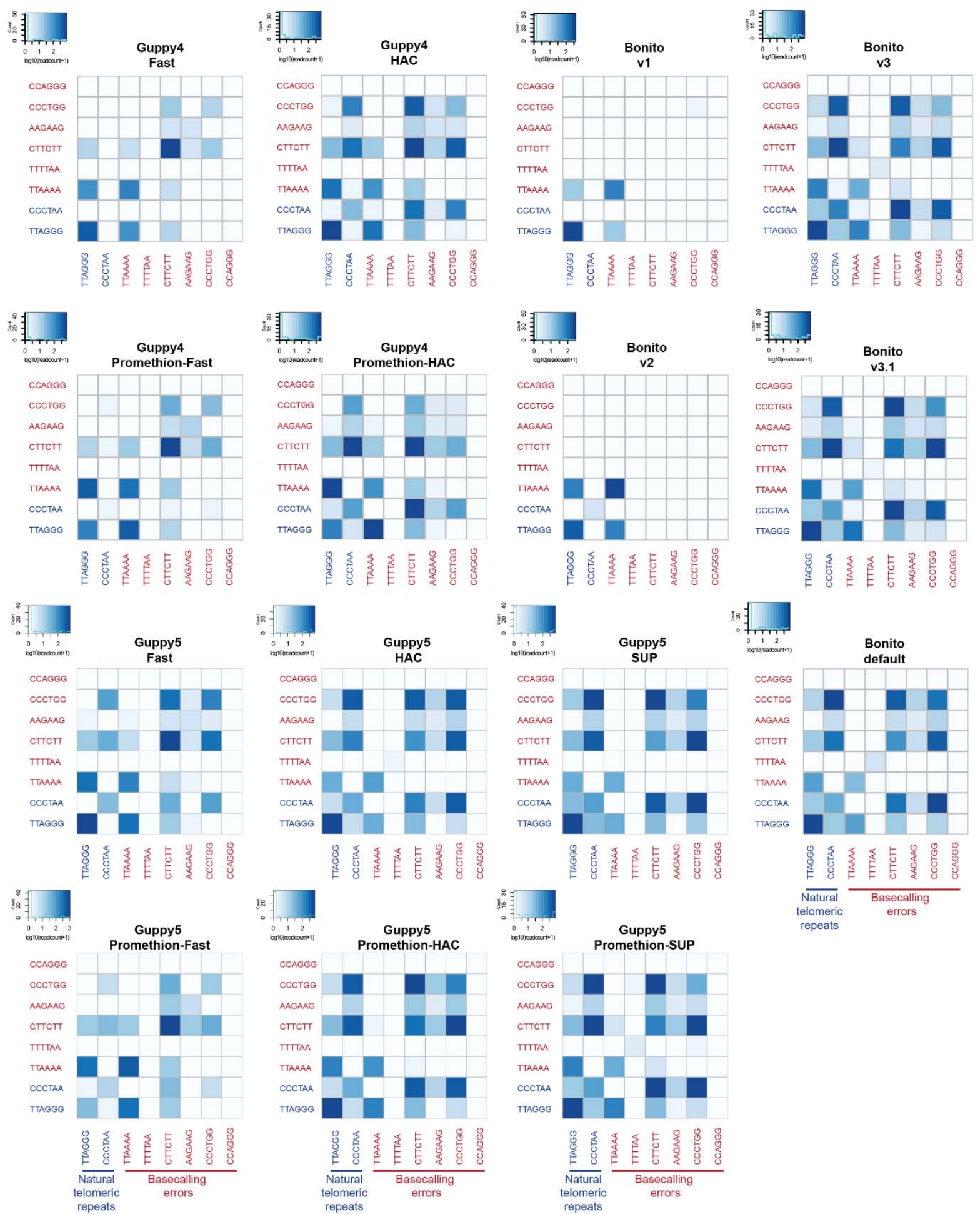
Nanopore promethion datasets. The frequency of telomeric repeats and basecalling artefacts observed on reads obtained are as depicted. 


\section{Supplementary Figure 7}
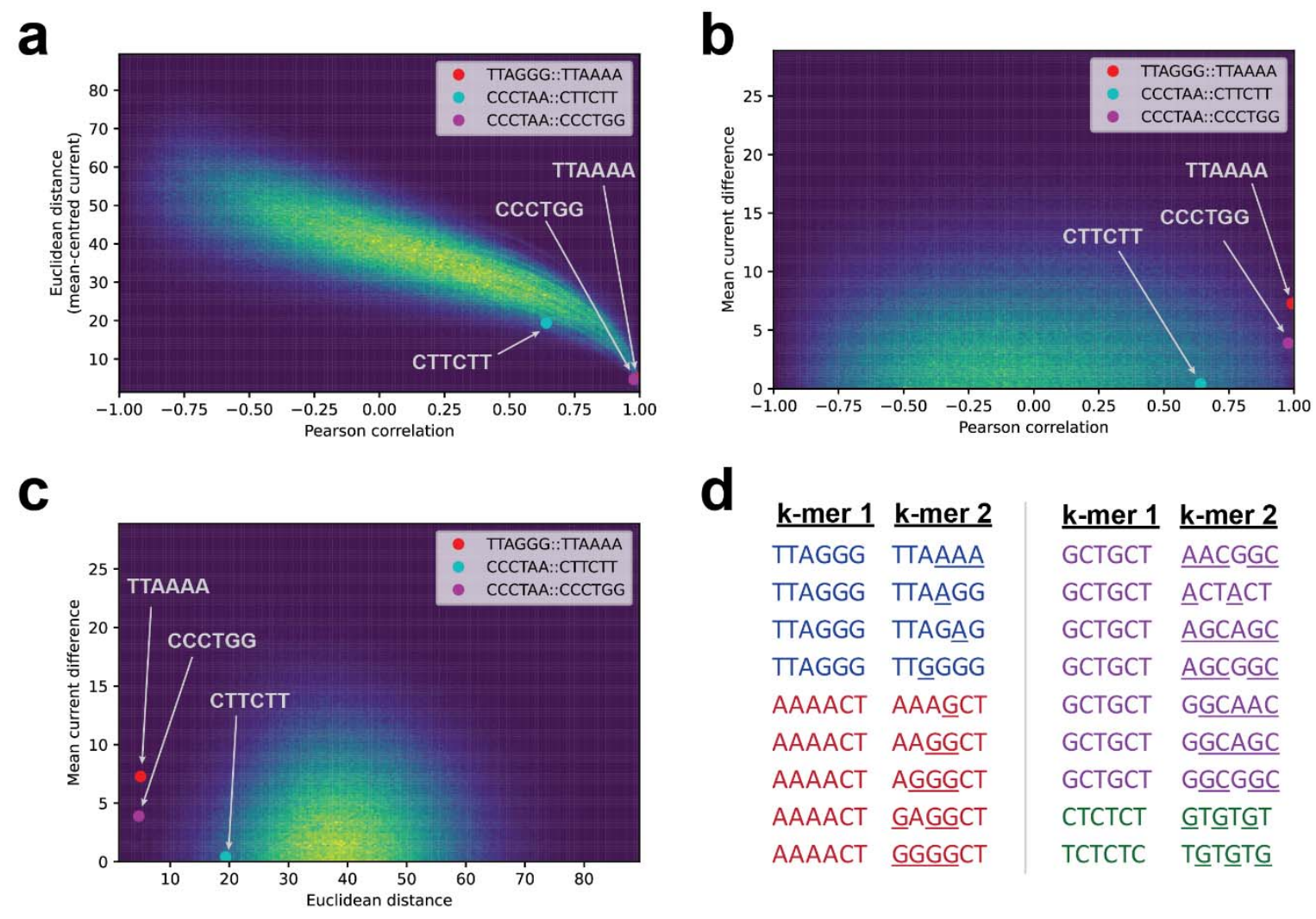

Q

\begin{tabular}{|c|c|c|c|}
\hline k-mer 1 & k-mer 2 & k-mer 1 & k-mer 2 \\
\hline TTAGGG & TТАAAA & GCTGCT & CGGG \\
\hline TTAGGG & TTAAGG & GCTGCT & ACTACT \\
\hline TTAGGG & TTAGAG & GCTGCT & GCAGC \\
\hline TTAGGG & TाGGGG & GCTGCT & $\underline{A G C G C}$ \\
\hline AAAACT & AAAGCT & GCTGCT & GGCAA \\
\hline AAAACT & $\mathrm{AAGG} C \mathrm{CT}$ & GCTGCT & $\mathrm{GGCAC}$ \\
\hline AAAACT & $\mathrm{AGGGCT}$ & GCTGCT & GGCGC \\
\hline AAAACT & $\underline{\mathrm{G}} \underline{\mathrm{GG}} \mathrm{G} T$ & СТСТСТ & $\underline{\text { GTGTG}}$ \\
\hline AAAACT & $\underline{\text { GGGGCT }}$ & ТСТстC & T $\underline{G}$ T $\underline{G} T$ \\
\hline
\end{tabular}

$\mathbf{e}$
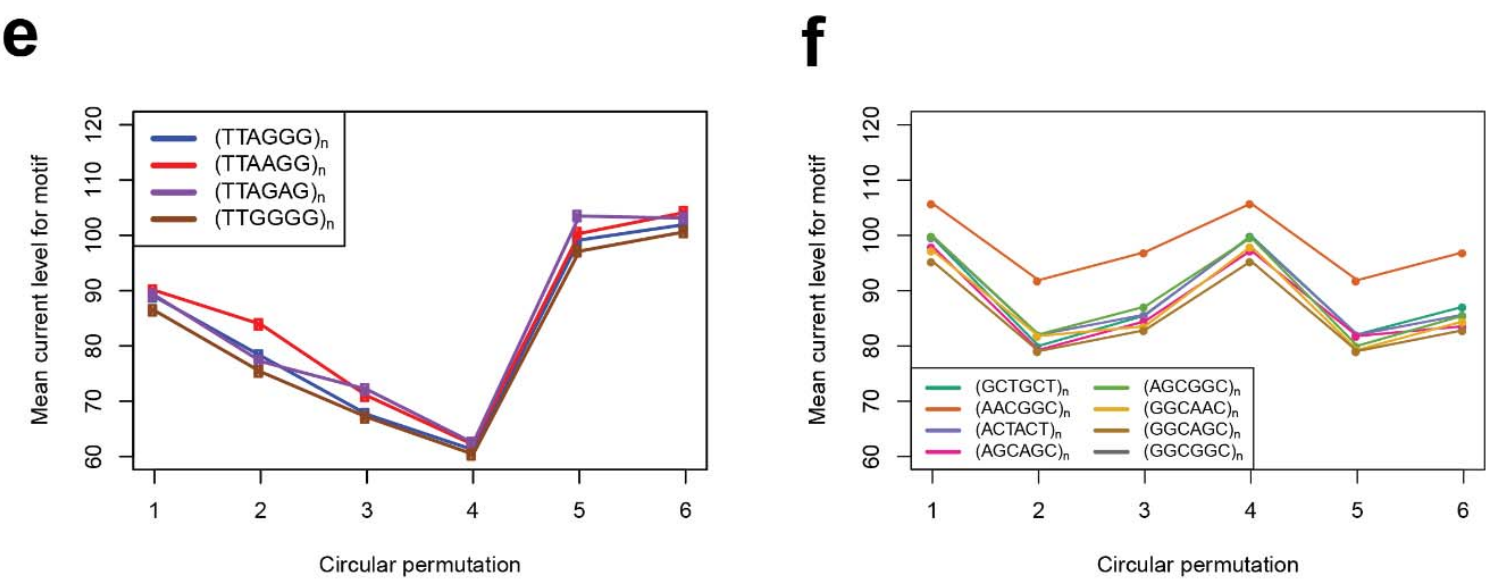

523 Supplementary Figure 7 Similarities between current profiles for all possible pairs of 6524 mer repeats. (a-c) Heatmaps depicting the Euclidean distances, Pearson correlation, and mean 525 current differences between current profiles between all possible 6-mer repeat sequences. 526 These are depicted as pairwise plots for (a) the Euclidean distances vs. the Pearson correlation, 527 (b) the mean current difference vs. the Pearson correlation, and (c) the mean current difference 528 vs. the Euclidean distance. The pairwise comparisons between the telomeric repeats and the 529 observed basecalling repeat artifacts are also highlighted in the plots. (d) Example pairs of k530 mer repeats with similar current profiles are as indicated. The nucleotides in k-mer 2 that differs 531 from k-mer 1 is underlined to highlight the nucleotides that differ between the two types of 
bioRxiv preprint doi: https://doi.org/10.1101/2022.01.11.475254; this version posted January 12,2022 . The copyright holder for this preprint (which was not certified by peer review) is the author/funder, who has granted bioRxiv a license to display the preprint in perpetuity. It is made available under aCC-BY-NC-ND 4.0 International license.

532 repeats. (e-f) Current profiles for repeats which were predicted to be highly similar to each other. 533 These are depicted for (e) TTAGGG telomeric repeats and telomere-like repeat sequences and 534 (f) GCTGCT repeat sequences that were highlighted in purple in Supplementary Figure 7d.

535

536 
bioRxiv preprint doi: https://doi.org/10.1101/2022.01.11.475254; this version posted January 12, 2022. The copyright holder for this preprint (which was not certified by peer review) is the author/funder, who has granted bioRxiv a license to display the preprint in perpetuity. It is made available under aCC-BY-NC-ND 4.0 International license.

\section{Supplementary Figure 8}

\section{a}

$>06 a 242 e 5-45 e 3-4 f 06-926 b-f 134 b 52 a 914 d$

GTGTGTGTGTGTGTGTGTGTGTGTGTGTGTGT GTGTGTGTGTGTGTGTGTGTGTGTGT GTGTGTGTGTGTGTGTGTGTGTGTGTGTGTGTGTGTGTGTGTGTGTGT GIGT GTGTGTIGTGTGTGTGTGGTGTGTGTGTGTTGTGTGTGTGTTGTGTGTGTGTGGTGTGTGTGTGTGGTGTGTGTGTIGTGTGTGTGTGGTGTGTGGT GTGTGTGT GTGTGTGTGTGIGTGTGTGTGTGTGTGTGTGTGTGIGTGTGTGTGTGTGIGTGTGTGTGTGTGTGTGTGTGTGTGTGTGTGTGTGTGTGTGTGTGTGT GTGTGTGTGTGTGTGTGTGTGTGTGT GTGTGTGTGTGTGTGTGTGTGTGTTGTGTGTGTGTGTGTGTGTGTGTGTGTGTGTGTGTGTGTGTGTGTGTGTGTGTGTG TGTGTGTGTGTGTGTGTGTGTGTGTGTGTGTGTGTGTGTTGTGTGTGTGTGTGTTGTGTGTGTGTGTGTGTGTGTGTGTGIGTGTGTGTGTGTGTGTGTGTGG TGTGTGTGTGTGTGTGTGTGTGTGTGTGTGTGTGTGTGGTGTGTGTGTGTGIGTGTGIGTGTGTGTGTGTGTGTGTGTGTGTGTGTGTGTGTGTGIGTGTGTGG TGTGTGTGTGTGTGT GTGTGTGTGTGTGTGTGTGTGTGTGTGTGTGTGTGTGTGTGTGTGTGTGTGTGTGTGTGTGTGTGTGTGTGTGTGTGTGTGTGTGTGTGTG TGTGTGTGTGTGTGTGTGTGTGTGTGTGTGTGTGTGTGTGTGTGTGTGTGTGTGTGTGTGTGTGTGTGTGTGTGTGTGTGTGTGTGTGTGTGTGTGTGTGTGTGTG

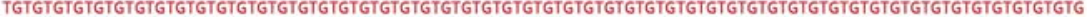

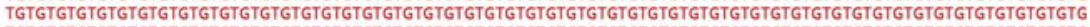

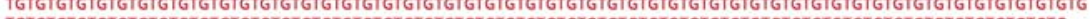
TGTGTGTGTGTGTGTGTGTGTGTGTGTGTGTGTGTGTGTGTTGTGTGTGTGGTGTGTGGTGTGTGTGTGTGTGTGTGTGTGTGTGTGTGTGTGTGTGTGTG

\section{b}

$>9504 e 9 d f-f d 60-4421-a 044-1 c 9553 c b 0879$

GTGTGGGTGGGTGTGTGTGTGTGTGTGTGTGTGTGTGTGTGTGTGTGTGTGTGTGTGTGTGTGTGTGTGTGTGTGTGTGTGTGT GTGTGTGTGTGTGTGTGTGTG (1) TGTGTGTGGTGTGTGGTGTGTGTGGTGTGTGTGGTGTGTGTGGTGTGTGGTGTGTGTGGTGTGTGTGGTGTGTGTGGGGTGTGGTGTGTGTGTG TGTGTGTGTGTGTGTGTGTGGGTGTGTGTGTGTGTGTGTGTGTGTGTGTGTGTGTGTGTGTGTGTGTGTGTGTGTGTGTGTGTGTGTGTGTGTGTGTGTGTGTGTG

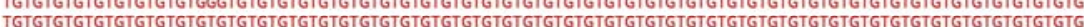
TGTGTGTGTGTGTGTGTGTGTGTGTGTGTGTGTGTGTGTGTGTGTGTGGTGTGTGTGTGTGTGTGTGTGTGTGTGTGGTGTGTGTGTGTGTGIGIGTGGG GTGGT TGTGTGTGTGTGTGTGTGTGTGTGTGTGTGTGTGTGT GTGTGTGTGTGTGTGTGTGTGTGTGTGTGTGTGTGTGTGTGTGTGTGIGTGTGTGTGTGTGTGTGTGTG TGTGTGTGTGTGTGTGTGTGTGTGTGTGTGTGTGTGTGTGTGTGTGTGTGTGTGTGTGTGTGTGTGTGTGTGTGTGTGTGTGTGTGTGTGTGTGTGTGTGTGTGTG

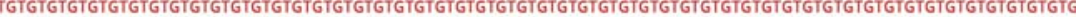

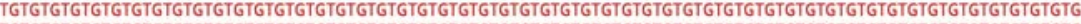
TGTGTGTGTGTGTGTGTGTGIGTGTGTGTTGTGTGTGTGTGTIGTGTGTGTGTGTITGTGTGTGTGTGTGTGTGTGTGTGTGTGTGTGTGT GTGTGTGTGTGTG

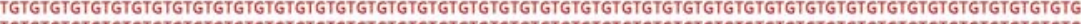

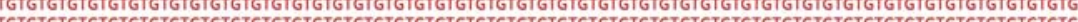

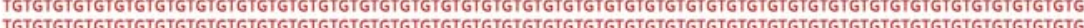
GTGGGTGGGGGGGGGGGGG

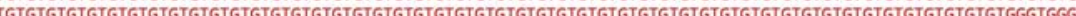
TGTGTGTGTGTGTGTGTGTGTGTGTGTGTGTGTGTGTGTGTGTGTGTGTGTGTGTGIGTGIGTGTGTGTGTGTGTGTGTGTGTGTGTGTGTGTGTGTGGTGTG

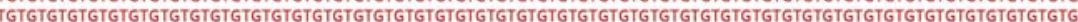
(1) TGGGTGGTGGTGTGTGTGTGTGTGTGT GTGTGTGTGTGTGTGTGTGTGTGTGTGTTGTGTGTGTGTGTGTGTGTGTGTGTGTGTGTGTGTGTGTGTGTGTTGT

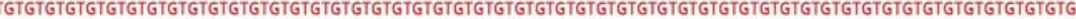

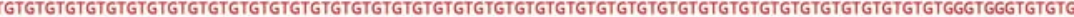

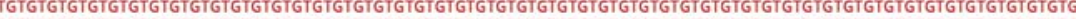
TGTGTGTGTGGTGTGTGTGTGGTGTGGTGTGTGTGGTGTGGTGTGTGTGGTGTGTGTGTIGTGTGTGTGGTGTGTGTGTGTGGTGTGGGGTGTGS

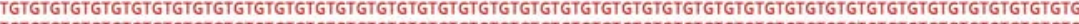
TGGGTGGGGGTGGGTGTGGGTGGGGGGGGTGGGGGGTGGGGGTGGGGGTGGGGGTGGGGGGG

C

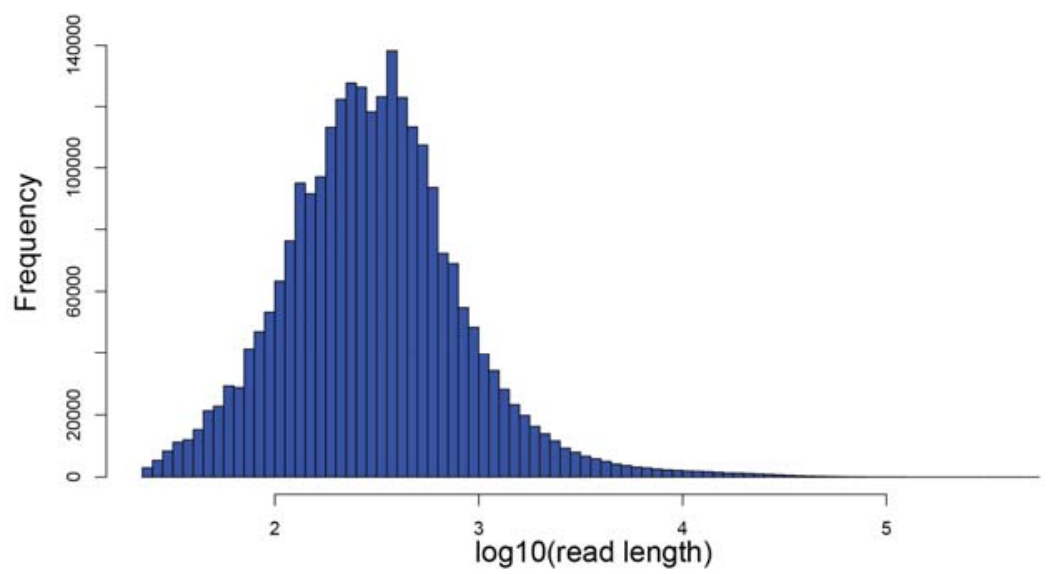
dataset. (a-b) Two representative reads from the $\mathrm{CHM} 13$ nanopore sequencing dataset with repeats $\geq 12$ ) in the $\mathrm{CHM} 13$ nanopore dataset. 


\section{Supplementary Figure 9}

a

\begin{tabular}{c|l} 
Bonito & $\begin{array}{l}\text { Forward } \\
\text { strand }\end{array}$ \\
(default) & $\begin{array}{l}\text { Reverse } \\
\text { strand }\end{array}$
\end{tabular}

\begin{tabular}{c|c} 
Bonito & $\begin{array}{l}\text { Forward } \\
\text { strand }\end{array}$ \\
(Tuned) & $\begin{array}{l}\text { Reverse } \\
\text { strand }\end{array}$
\end{tabular}

b

Bonito

(default)

\begin{tabular}{c|l} 
Bonito & $\begin{array}{l}\text { Forward } \\
\text { strand }\end{array}$ \\
(Tuned) & $\begin{array}{l}\text { Reverse } \\
\text { strand }\end{array}$
\end{tabular}

C

Bonito
(default)

\begin{tabular}{c|l} 
Bonito & $\begin{array}{l}\text { Forward } \\
\text { strand } \\
\text { (Tuned) } \\
\begin{array}{l}\text { Reverse } \\
\text { strand }\end{array}\end{array}$
\end{tabular}

Forward

strand

Reverse strand

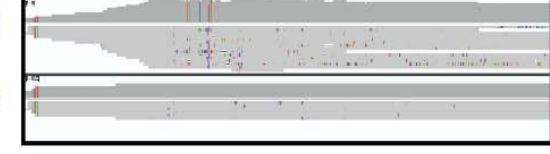

Training data
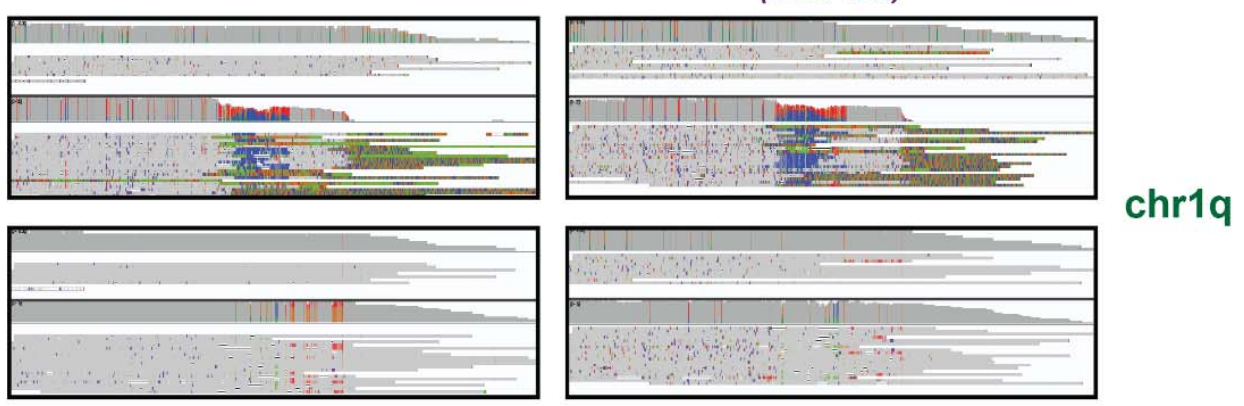

Testing data

(held out)
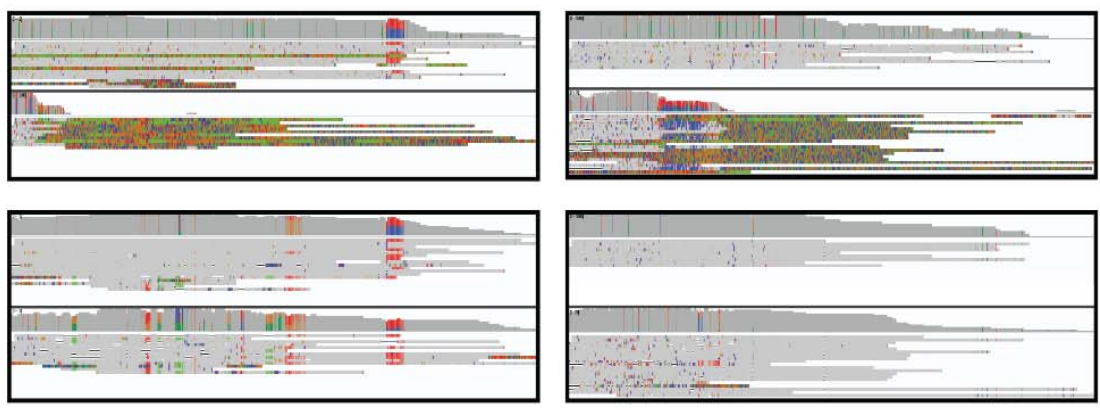

chr3q
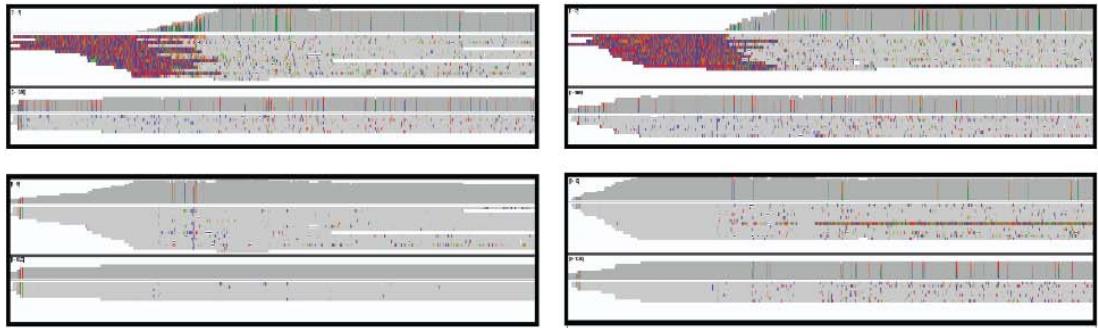

chr11p d

Bonito

(default)

Forward

strand

Reverse

strand

\begin{tabular}{c|l} 
Bonito & $\begin{array}{l}\text { Forward } \\
\text { strand }\end{array}$ \\
(Tuned) & $\begin{array}{l}\text { Reverse } \\
\text { strand }\end{array}$
\end{tabular}
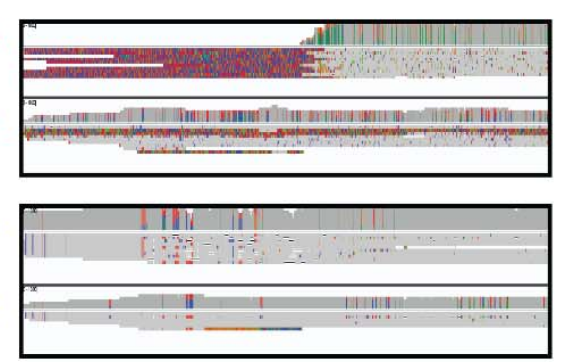
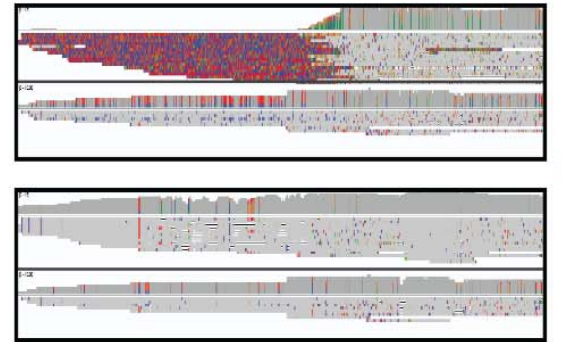

chr12p

543

544

545

546

547
Supplementary Figure 9 Additional examples for the performance of the tuned bonito basecaller on telomeres on other chromosomal arms. The tuned model was applied to the training dataset used for model training, and on an additional held out test dataset that was not used during model training. IGV screenshots of the default and tuned bonito basecaller on the 
bioRxiv preprint doi: https://doi.org/10.1101/2022.01.11.475254; this version posted January 12,2022 . The copyright holder for this preprint (which was not certified by peer review) is the author/funder, who has granted bioRxiv a license to display the preprint in perpetuity. It is made available under aCC-BY-NC-ND 4.0 International license.

548 training and testing dataset for the chromosomal arms (a) chr1q, (b) chr3q, (c) chr11p and (d) 549 chr12p are as depicted. Related to Figure $2 b$.

550

551 


\section{Supplementary Figure 10}
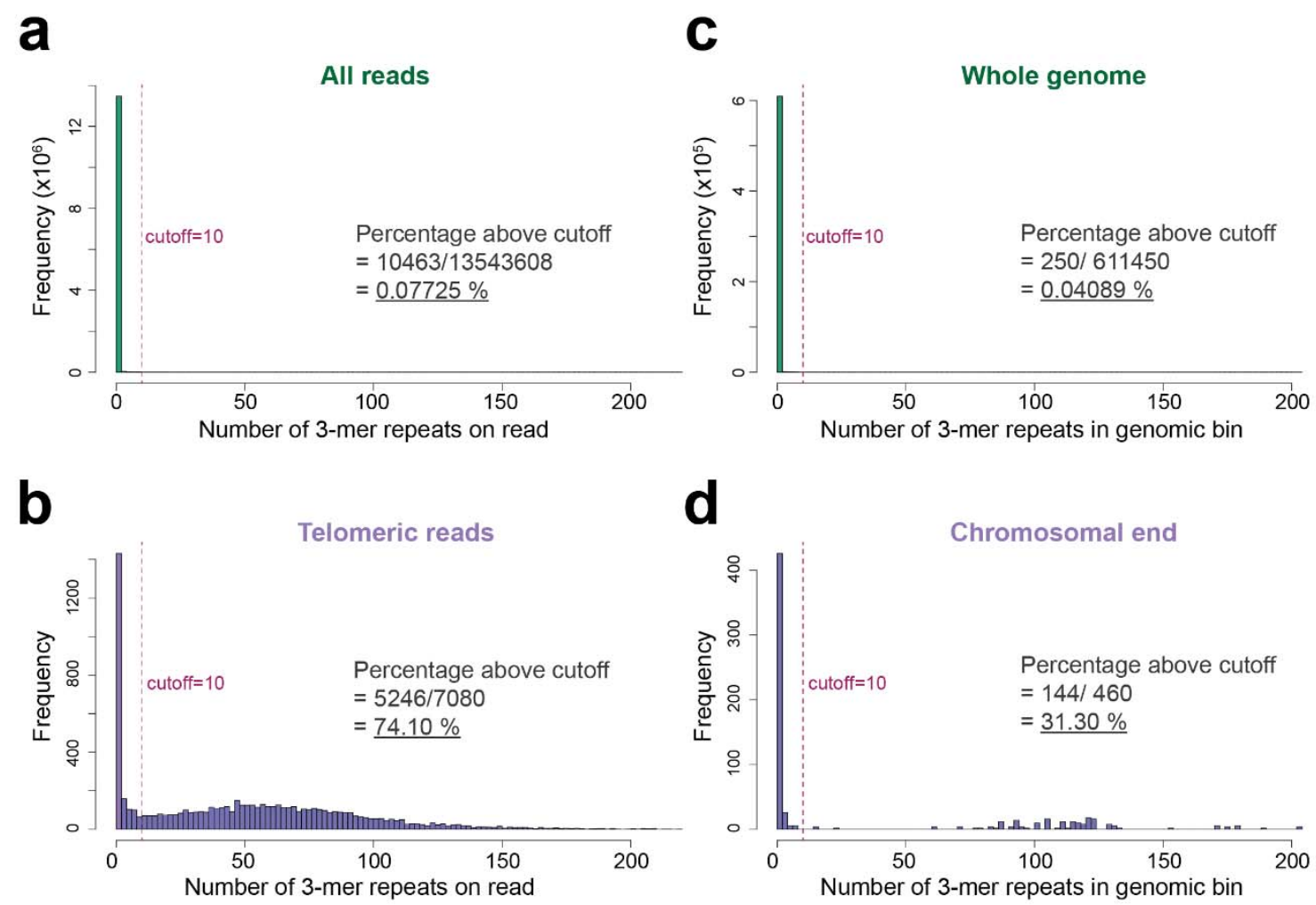
reads at telomeres and on reads found at the rest of the genome in the CHM13 dataset. (a-b) The sum of 3-mer telomeric repeats $\left[\left(\mathrm{TTAGGG}_{3}\right)_{3}(\mathrm{CCCTAA})_{3}\right]_{3}$ and basecalling error repeats [(TTAAAA $)_{3},(\text { TTTTAA })_{3},(\text { CTTCTT })_{3},(\text { AAGAAG })_{3},(\text { CCCTGG })_{3},(\text { CCAGGG })_{3}$ ] on (a-b) each long-read or (c-d) genomic bin are as depicted on the $x$-axis of each histogram. The histograms represent the frequency of these repeats on (a) all long-reads in the CHM13 dataset, (b) telomeric reads in the CHM13 dataset, (c) $20 \mathrm{~kb}$ genomic bins with $10 \mathrm{~kb}$ moving window for the full $\mathrm{CHM} 13$ reference genome, (d) and for the 10 genomics bins on each chromosomal end of the CHM13 genome. 
bioRxiv preprint doi: https://doi.org/10.1101/2022.01.11.475254; this version posted January 12,2022 . The copyright holder for this preprint (which was not certified by peer review) is the author/funder, who has granted bioRxiv a license to display the preprint in perpetuity. It is made available under aCC-BY-NC-ND 4.0 International license.

\section{Supplementary Tables}

565 pearson correlation, Euclidean distance, and mean current difference between each pair of kmer is as presented in the table. 